\title{
ON KERNEL REPRESENTATION OF LINEAR OPERATORS
}

BY

\section{DOROTHY MAHARAM}

1. Introduction. In a previous paper [6] a study was made of " $F^{\prime}$ integrals," that is (roughly speaking) of linear, countably additive orderpreserving mappings $\phi$ from one (real) function space, $F$, to another, $F^{\prime}$. It was shown that (assuming, as we do throughout, that $F$ and $F^{\prime}$ satisfy the countable chain condition) every such $\phi$ can be obtained, to within an isomorphism of $F$, by coordinatewise integration in a product space. That is, we may regard $F$ as imbedded as a subspace in the space of (suitably defined) "measurable" functions, modulo "null" sets, on the direct product $J \times Y$ of two spaces, of which $J$ has a $\sigma$-finite numerical measure $m$, in such a way that for all "integrable" $f \in F$ we have

$$
\phi(f)=g \in F^{\prime} \quad \text { where } \quad g(y)=\int_{J} f(x, y) d m(x) .
$$

Now if $k$ is a given non-negative finite "measurable" function on $J \times Y$, another $F^{\prime}$-integral $\psi$ can be defined on $F$ by the rule

$$
\psi(f)=h \in F^{\prime}, \text { where } h(y)=\int_{J} k(x, y) f(x, y) d m(x) .
$$

That is, $\psi(f)=\phi(k f)$, for all suitable $f \in F$. Here $k$ need not be in the subspace $F\left({ }^{1}\right)$. The main problem discussed in this paper is the question of under what circumstances two given $F^{\prime}$-integrals $\phi, \psi$, on a given function space $F$, can be put in this sort of relation. The problem can be formulated in two ways. Firstly, we may ask when a fixed $k \in F$ exists such that $\psi(f)=\phi(k f)$ identically; if this is the case, we say that $\psi$ has a "kernel representation" in terms of $\phi$, with kernel $k$. We shall see ( $\$ 4)$ that this question, which amounts to requiring that $\psi$ be in a sense "differentiable" with respect to $\phi$, has a simple answer if $\phi$ is "full-valued" (cf. 2.3 below). Secondly, we may ask when $\phi$ and $\psi$ can, as in the above example, be extended to $F^{\prime}$-integrals $\phi^{*}, \psi^{*}$ defined on some function space $F^{*} \supset F$, in such a way that $\psi^{*}$ has a kernel representation in terms of $\phi^{*}$ (with kernel in $F^{*}$ ). If this is the case, we say that $\psi$ has an "extended kernel representation" in terms of $\phi$. We shall obtain

Presented to the Society, October 30,1954; received by the editors July 7, 1954.

(1) For example, the "kernel operators" usual in analysis have the form $h(y)=\int k(x, y) f(x) d x$. This is the special case of (2) in which $f(x, y)$ is independent of $y$. Here $F$ consists of those functions on $J \times Y$ which are independent of $y ; F$ is isomorphic, in a natural way, to the space of (measurable) functions on $J$. 
necessary and sufficient conditions on $\phi$ and $\psi$ for this to be possible (Theorem $7, \S 5)$; they amount to saying that $\phi$ "dominates" $\psi$ in a certain sense (5.3). The solution to this second problem is deduced from that of the first; we show that $\phi$ has a full-valued extension $\phi^{*}$ which is "minimal" in the sense that $F^{*}$ is as small as possible, and that it suffices to consider this minimal $F^{*}$ as the extension space involved.

So far, the kernels contemplated have all been non-negative. But a linear, countably additive mapping $\psi$ of $F$ in $F^{\prime}$, or " $F^{\prime}$-operator on $F$ " is still provided by (2) even if $k$ can change sign; and we conclude by applying the previous theory to determine just which $F^{\prime}$-operators on $F$ can be put in the form (2), with arbitrary (finite) kernels. If $\psi$ is expressible in this way, then $\psi$ is clearly of "bounded variation" in a natural sense $\left({ }^{2}\right)$, for we may write $\psi=\psi^{+}-\psi^{-}$where $\psi^{+}(f)=\int k^{+}(x, y) f(x, y) d m(x), \psi^{-}(f)=\int k^{-}(x, y) f(x, y) d m(x)$, $k^{+}$and $k^{-}$denoting the positive and negative parts of $k$. We shall show that, conversely, every $F^{\prime}$-operator on $F$ which is of bounded variation can, after an isomorphism of $F$, be put in the form (2) with a suitable (finite, but no longer positive) kernel. This amounts to representing both the positive and negative parts of $\psi$ in this form simultaneously (each with a non-negative kernel); and this simultaneous representation is achieved by constructing a common "dominant" $\phi$ for them, and representing $\phi$ in the form (1).

A further extension is possible. We are concerned in this paper only with real function spaces, but there would be no difficulty in extending the results to complex function spaces. The $F^{\prime}$-operator $\psi$ could then be expressed as $\psi_{1}+i \psi_{2}$, where $\psi_{1}$ and $\psi_{2}$ are real for real functions; and $\psi$ is of bounded variation if and only if $\psi_{1}$ and $\psi_{2}$ are, so that the problem of representing $\psi$ comes to that of representing four (real) positive $F^{\prime}$-operators simultaneously. In fact, the construction in 5.8 below gives a common dominant for countably many $F^{\prime}$-integrals at once, so that we can represent countably many " $F^{\prime}$ operators of bounded variation" (real or complex) in the form (2) simultaneously.

This representation theorem is related to work of Kantorovitch and Vulich $[2 ; 3]$, and our $F^{\prime}$-operators of bounded variation are similar to their "regular operators." Kantorovitch and Vulich assume the countable chain condition (in effect) only "locally," and their function spaces need not have a unit function; apart from this, the present set-up is more general than theirs. The methods and results are quite different, since these authors were mainly concerned with special cases, in most of which norms are available.

The foregoing description of the present theory is a little over-simplified, and our results are somewhat more general than those stated. A "function

(2) That is, $\psi$ is the difference between two positive (order-preserving) operators, i.e., essentially the difference between two $F^{\prime}$-integrals. The definition adopted below (6.1), though different, is substantially equivalent to this (see Theorem 10,6.3). 
space" $F$ (cf. [6] and 2.1 below) includes infinite functions, so the operators considered are to be defined, not necessarily on all of $F$, but merely on sufficiently "dense" linear subspaces $G$ of $F$. (The precise formulations are given in 2.2 and 6.1 below.) Further, the "positive $F^{\prime}$-operators" we consider are a little more general than the " $F^{\prime}$-integrals" of [6], as two normalising conditions are dropped $(2.2,6.2)$; these normalising conditions were convenient when only one $F^{\prime}$-integral was considered, but become undesirable when, as here, we have to deal with several $F^{\prime}$-integrals simultaneously.

The theory applies both to linear transformations of a function space in itself (when $F^{\prime}=F$ ), and to linear functionals (when $F^{\prime}=$ real numbers = space of functions on a single point). Thus many of our results constitute generalizations of results well known for linear functionals; for example, the solution of the first kernel representation problem includes the Radon-Nikodym theorem (Theorem 5, 4.3). The theory of the minimal full-valued extension of $\phi$ (§3), on which our methods are based, is however a new feature, for every linear functional is automatically full-valued. As by-products, we obtain several other results which may be of independent interest, for example, a uniqueness theorem for extensions of $F^{\prime}$-measures (3.3), and a rather strong form of the Hahn decomposition theorem for (real) $F^{\prime}$-operators of bounded variation (6.5).

\section{Preliminaries.}

2.1 Notation. In general we use the same notation as in [6], an acquaintance which with is assumed. Here we recall that a "function space" $F=F(S, \mathbb{B}, \mathcal{N})$ is determined by a set $S$, a Borel field $\mathrm{B}$ of "measurable" subsets of $S$, and a $\sigma$-ideal $N \subset B$ of "null" sets (though no actual measure is assumed); $F$ consists of the equivalence classes modulo $\mathcal{N}$ of $\mathbb{B}$-measurable functions on $S$ whose values are real numbers or $\pm \infty . F$ is in fact determined, to within strict isomorphism [6, p. 159], by the Boolean $\sigma$-algebra $E=B / \mathcal{N}$, and we also write $F$ as $F(E)$. We usually do not distinguish between a $\mathcal{B}$-measurable function $f$ on $S$ and its equivalence class, $\{f\}$, modulo $\mathcal{N}$; the latter, of course, is the typical element of $F$. Similarly we shall not distinguish between a set $x \in \mathcal{B}$ and its equivalence class $\{x\} \in E$. All the function spaces considered are to satisfy the countable chain condition [6, p. 160]. We make the conventions that $0 \cdot \infty=\infty \cdot 0=0$, but that $\infty-\infty$ is not defined; thus $F$ is not quite a linear space, though the finite elements of $F$ form a linear space $\left.{ }^{3}\right)$.

If $f, g \in F$ and $x \in E$ (or $\mathcal{B}$ ), we write " $f \leqq g$ on $x$ " to mean that $f(s) \leqq g(s)$ for each $s \in x-N$, where $N \in \mathcal{N}$. Similarly " $f \ll g$ on $x$ " means $f(s)<g(s)$ for each $s \in x-N(N \in \mathcal{N})$. If $x$ can be taken to be the unit element of $E$ here (corresponding to the whole set $S$ ), we write simply $f \leqq g$ or $f \ll g$. The notation " $f<g$ " means $f \leqq g$ and $f \neq g$. If $f_{n} \in F(n=1,2, \cdots)$, the elements

( $\left.{ }^{3}\right)$ The corresponding theory for operators $\phi$ which map one linear space in another (or, more generally, map one vector $\sigma$-lattice, satisfying the countable chain condition, in another) is included in the present setting; cf. [6, p. 156]. 
$\sup f_{n}$, inf $f_{n}$, and (if they exist) $\sum f_{n}$ and $\lim f_{n}$ are similarly defined pointwise (modulo $\mathcal{N}$ ). (They may be infinite.) In view of the countable chain condition, $\sup f_{\alpha}$ and inf $f_{\alpha}$ exist (possibly infinite) for arbitrary collections $\left\{f_{\alpha}\right\}$ of elements of $F$, but in general they cannot be defined pointwise. We write $\sup (f, 0)=f^{+}, \inf (f, 0)=-f^{-} ;$thus $f^{+} \geqq 0, f^{-} \geqq 0, f^{+}-f^{-}$exists and equals $f$, and $f^{+}+f^{-}=|f|$, the function (modulo $\mathcal{N}$ ) whose value at each $s \in S$ is $|f(s)|$.

For each $x \in E, E(x)$ denotes the principal ideal consisting of all $y \in E$ such that $y \leqq x$; it corresponds to the "subspace" $x$ of $S$. We regard the function space $F(E(x)$ ) as imbedded in $F(E)$ in an obvious way, a (B-measurable) function $g$ on $x(\bmod \mathcal{N})$ corresponding to the function on $S$ which agrees with $g$ on $x$ and is 0 outside $x$. Similarly if $E_{1}$ is a sub-algebra of $E$ (corresponding to a Borel field $\left.\mathcal{B}_{1} \subset B\right), F\left(E_{1}\right)$ can be regarded as naturally imbedded in $F(E)$, the $\mathbb{B}_{1}$-measurable function $f \in F\left(E_{1}\right)$ corresponding to itself. For any set $A \subset E, \mathbb{B}(A: E)$ denotes the smallest $\sigma$-algebra $B$ such that $A \subset B \subset E$; it corresponds $(\bmod \mathcal{N})$ to the Borel field generated by the sets corresponding to the elements of $A$. Usually it is clear which $E$ is meant, and we write $\mathrm{B}(A: E)$ simply as $\mathrm{B}(A)$.

The unit and zero elements of $E$ are written as $e, o$ respectively; if $E^{\prime}, E^{1}$, etc. are Boolean $\sigma$-algebras, we write their unit elements as $e^{\prime}, e^{1}$, etc., without further comment. (It is usually not necessary to distinguish between their zero elements.) The characteristic function $(\bmod \mathcal{N})$ of $x \in E$ is denoted by $\chi(x)(\in F)$. Thus $\chi(e)=1$ (i.e., the constant function whose value at each $s \in S$ is 1$)$, and $\chi(o)=0$. For each $f \in F$, the locus of $f$, written [f], is the element of $E$ corresponding to the subset $\{s \mid f(s) \neq 0\}$ of $S$. Thus, for all $f \in F$, we have $|f| \leqq \infty \cdot \chi[f]$; here, and in similar expressions, we write $\chi[f]$ instead of $\chi([f])$, to simplify the notation.

Finally, the set of non-negative elements of $F$ will be denoted by $F^{+}$; and, generally, if $G \subset F$, we write $G \cap F^{4}$ as $G^{+}$.

$2.2 F^{\prime}$-integrals and measures; relaxation. Let $F=F(S, B, \mathcal{N})=F(E)$ and $F^{\prime}=F\left(S^{\prime}, \mathbb{B}^{\prime}, \mathcal{N}^{\prime}\right)=F\left(E^{\prime}\right)$ be two function spaces (possibly the same). An $F^{\prime}$-integral on $F$ is, in the first instance, a mapping $\phi$ of $F^{+}$in $F^{\prime+}$ such that $[6$, p. 161]:

$(\alpha) \phi\left(\sum f_{n}\right)=\sum \phi\left(f_{n}\right)\left(f_{n} \in F^{+} ; n=1,2, \cdots\right)$.

(B) There exist $g_{n} \in F^{+}(n=1,2, \cdots)$ such that $\sum g_{n} \gg 0$ and $\phi\left(g_{n}\right) \ll \infty$.

$(\gamma) \phi(1) \gg 0$.

(ס) If $f>0, \phi(f)>0$.

It was shown in [6] that each such mapping $\phi$ can be extended uniquely, in a natural way, to a set $G=\left\{f \mid f \in F\right.$ and $\phi\left(f^{+}\right)-\phi\left(f^{-}\right)$exists $\}$of "integrable" functions, and we shall usually suppose that this extension has been carried out. $\phi$ is then linear on $G$, in the sense that $\phi(a f+b g)=a \phi(f)+b \phi(g)$ whenever $a, b$ are real numbers and both sides have a meaning. 
For any $F^{\prime}$-integral $\phi$ on $F$, we write

$$
\lambda(x)=\phi(\chi(x))
$$

and have that $\lambda$ is a mapping of $E$ in $F^{\prime+}$ such that:

(a) If $x_{m} x_{n}=o\left(m \neq n, m, n=1,2, \cdots, x_{m} \in E\right)$, then $\lambda\left(\sup x_{m}\right)=\sum \lambda\left(x_{m}\right)$.

(b) $e=\sup a_{n}\left(a_{n} \in E, n=1,2, \cdots\right)$, where $\lambda\left(a_{n}\right) \ll \infty$.

(c) $\lambda(e) \gg 0$.

(d) If $x \neq 0$, then $\lambda(x)>0$.

Any mapping $\lambda$ of $E$ in $F^{\prime+}$ satisfying conditions (a) $-\left(\right.$ d) is called an $F^{\prime}$ measure on $E$. Any such $\lambda$ determines uniquely a corresponding $F^{\prime}$-integral $\phi$, by a process analogous to ordinary Lebesgue integration [6, p. 164], and we write

$$
\phi(f)=\int f d \lambda \quad\left(f \in F^{+}, \text {or more generally } f \in G\right) .
$$

The conditions $(\gamma)$ and $(\delta)$ on $\phi$, and (c) and (d) on $\lambda$, are in the nature of "normalising" conditions, and we shall later $(\$ \$ 4-6)$ have to consider situations in which they are not satisfied. Hence we define a mapping $\phi$ of $F^{+}$in $F^{\prime+}$ to be a relaxed $F^{\prime}$-integral on $F$ if it satisfies $(\alpha)$ and $(\beta)$ above; similarly a relaxed $F^{\prime}$-measure on $E$ is a mapping $\lambda$ of $E$ in $F^{\prime+}$ satisfying (a) and (b). All the preceding remarks continue to apply; a relaxed $F^{\prime}$-integral $\phi$ can be uniquely extended so as to be linear on the set $G$ defined above, and corresponding relaxed $F^{\prime}$-integrals and measures determine each other in accordance with (1) and (2). For emphasis, $F^{\prime}$-integrals and measures which satisfy $(\gamma),(\delta)$ and (c), (d) will sometimes be called "strict"; however, all $F^{\prime}$-integrals and measures are strict unless the contrary is stated.

2.3 Full-valued $F^{\prime}$-integrals. As in [6, p. 174], an $F^{\prime}$-integral $\phi$ on $F$ is called full-valued if it has the property $\left.{ }^{4}\right)$

(1) Given $f \in F^{+}$and $g^{\prime} \in F^{\prime+}$ such that $g^{\prime} \leqq \phi(f)$, there exists $g \in F^{+}$such that $g \leqq f$ and $\phi(g)=g^{\prime}$.

The functions appearing here may be infinite; but is is easy to see that the meaning of (1) is not changed if we require $f$ and $\phi(f)$ (and thus also $g$ and $\left.g^{\prime}\right)$ to be finite. Every numerical $F^{\prime}$-integral (the case in which $F^{\prime}$ reduces to the functions on a single point) is automatically full-valued.

A necessary and sufficient condition for a (strict) $F^{\prime}$-integral $\phi$ on $F$ to be full-valued is $[6$, p. 174]:

(2) $E^{\prime}$ is $(\sigma-)$ isomorphic to a sub-algebra $A$ of $E$ in such a way that, if $a \in A$ corresponds to $a^{\prime} \in E^{\prime}$ in this isomorphism, then $\lambda(x a)=\lambda(x) \chi\left(a^{\prime}\right)$ for all $x \in E, \lambda$ being the $F^{\prime}$-measure corresponding to $\phi$.

(4) Note that the similar requirement for $\lambda$ is distinct (it is, in fact, stronger); see [6, p. 174]. Throughout the present paper, "full-valued" refers to $\phi$, not to $\lambda$. 
2.4 Extensions. Suppose $E$ is $(\sigma$-)isomorphic to a sub-algebra $A$ of a Boolean $\sigma$-algebra $E^{*}$, and that $\phi$ is a given $F^{\prime}$-integral on $E$. An $F^{\prime}$-integral $\phi^{*}$ on $F\left(E^{*}\right)$ is said to be an extension of $\phi$ if $\phi^{*}$ agrees with $\phi$ on $F(A)$, considered as a subspace of $F\left(E^{*}\right)$, i.e., if for each $g \in F(A)^{+}$, corresponding to $f \in F(E)$, we have $\phi^{*}(g)=\phi(f)$. It is obviously equivalent to require of the corresponding $F^{\prime}$-measures $\lambda, \lambda^{*}$ that if $a \in A$ corresponds to $x \in E$, then $\lambda^{*}(a)=\lambda(x)$; in other words, the isomorphism between $E$ and $A$ is to be a "strict $F^{\prime}$-isomorphism" in the sense of $\left[6\right.$, p. 166], i.e., preserves $F^{\prime}$-measures.

In $[6, \S 5]$, a construction was given showing that every $F^{\prime}$-integral on $F(E)$ has a full-valued extension $\left({ }^{5}\right)$. In the next section we shall consider this construction (slightly modified) in more detail, showing that it leads to a minimal full-valued extension, and deriving other properties which will be needed later.

3. The product $(E, \lambda) \times E^{\prime}$.

3.1 The construction. Let $\lambda$ be an $F^{\prime}$-measure on $E$, where $F^{\prime}=F(E)$. It was shown in [6, pp. 169-172] that another $F^{\prime}$-measure algebra, the "direct product" $(E, \lambda) \times E^{\prime}$, can be defined as follows $\left(^{6}\right)$. In the product $R \times S^{\prime}$ of the representation spaces of $E$ and $E^{\prime}$, an "outer $F^{\prime}$-measure" $\lambda^{*}$ can be constructed which has the property that for each "rectangle" $x \times x^{\prime}(x \in E$, $\left.x^{\prime} \in E^{\prime}\right), \lambda^{*}\left(x \times x^{\prime}\right)=\lambda(x) \chi\left(x^{\prime}\right) . \lambda^{*}$ is $\sigma$-additive on a certain field $\mathcal{B}^{*}$ of "measurable" sets (which includes all "rectangles"), and vanishes on an ideal $\mathcal{N}^{*}$ of "null" sets. The Boolean $\sigma$-algebra $E^{*}=\mathscr{B}^{*} / \mathcal{N}^{*}$ is then the desired product $(E, \lambda) \times E^{\prime}$, and $\lambda^{*}$ is the $F^{\prime}$-measure on it. Clearly $E^{\prime}$ is isomorphic to a sub-algebra $E_{0}^{\prime}$ of $E^{*}$, the element $x^{\prime}$ of $E^{\prime}$ corresponding to the "cylinder" $e \times x^{\prime}$. Similarly $E$ is isomorphic to a sub-algebra $E_{0}$ of $E^{*}$, the element $x \in E$ corresponding to the "cylinder" $x \times e^{\prime}$; notice that $\lambda^{*}$ then agrees with $\lambda$ on $E$. Thus, if $\phi$ is the $F^{\prime}$-integral on $F(E)$ corresponding to $\lambda$ (i.e., $\phi(f)$ $\left.=\int f d \lambda\right)$, and if $\phi^{*}$ similarly corresponds to $\lambda^{*}$ on $F\left(E^{*}\right), \phi^{*}$ is an extension of $\phi$; and, from $2.3(2), \phi^{*}$ is full-valued. For brevity we shall sometimes refer to the $\phi^{*}$ and $\lambda^{*}$ so constructed as the "standard" $F^{\prime}$-integral and $F^{\prime}$-measure on $F\left(E^{*}\right)$ and $E^{*}$, respectively.

In $[6$, pp. 170,171$]$ it was further shown that any $H \in B^{*}$ can be written as $U H_{n}(n=1,2, \cdots)$ where $H_{n}$ is contained in a "cylinder" of the form $a_{n} \times e^{\prime}$ where $\lambda\left(a_{n}\right) \ll \infty$; and that for each $H_{n}$ there exists a double sequence of "rectangles" $r_{n m k}$ such that $\bigcup_{m} r_{n m k} \supset H_{n}$ and $\lambda^{*}\left(\bigcup_{m} r_{n m k}-H_{n}\right) \leqq 1 / k$. It

(5) In fact, the extension $\phi^{*}$ constructed in [6] has the stronger property that $\lambda^{*}$ is fullvalued. However, this is in general a larger extension than we require here.

${ }^{(6)}$ In [6] this construction was applied, not to $(E, \lambda)$, but to an auxiliary $F^{\prime}$-measure algebra $(P, L)$ which had an additional property (loc. cit. p. 169, equation (1)). This property, however, is irrelevant to the results which are quoted here. Note that the construction of $(E, \lambda) \times E^{\prime}$ uses the representation space realizations of $E$ and $E^{\prime}$. It is an open question whether arbitrary realizations of $E$ and $E^{\prime}$ could be used (with the same construction), though the answer is affirmative in some cases (for example, if $E^{\prime}$ is derived from a numerical measure space). 
follows that, in $E^{*}, H^{*}=\cup_{n} \cap_{k} \bigcup_{m} r_{n m k}$, so that the Borel field $\mathscr{B}\left(E_{0} \cup E_{0}^{\prime}: E^{*}\right)$ generated by $E_{0}$ and $E_{0}^{\prime}$ (in $E^{*}$ ) is the whole of $E^{*}$.

We shall see later (Theorem 2, 3.5) that the properties of $(E, \lambda) \times E^{\prime}$ which have been mentioned here suffice to characterize it completely, to within an $F^{\prime}$-measure-preserving isomorphism.

3.2 Other products. The type of product just discussed includes several other products as special cases. The product of a numerical measure algebra $(J, \mu)$ and an arbitrary $\sigma$-algebra $E^{\prime}$ (see $[4, \S 4]$ ) is included, for we can regard the number $\mu(x)(x \in J)$ as a constant function over $E^{\prime}$, so that $\mu$ becomes an $F^{\prime}$-measure on $J$; the resulting product $(J, \mu) \times E^{\prime}$ then coincides with that in [4], as follows from the characterization given in [4]. The ordinary direct product of two numerical measure algebras, say $\left(E_{1}, \lambda_{1}\right)$ $\times\left(E_{2}, \lambda_{2}\right)$, can then be derived from the product $\left(E^{*}, \lambda^{*}\right)=\left(E_{1}, \lambda_{1}\right) \times E_{2}$ (an $F\left(E_{2}\right)$-measure algebra) by integration, the numerical measure of any element $x \in E^{*}$ being $\int \lambda^{*}(x) d \lambda_{2}$; see [5, §2]. More generally, the product of a numerical measure algebra $(J, \mu)$ and a general $F^{\prime}$-measure algebra $(E, \lambda)$ (cf. the construction of $(P, L)$ in [6, p. 169]) can be defined by integration starting from $(J, \mu) \times E=\left(J^{*}, \mu^{*}\right)$ say, the final $F^{\prime}$-measure of $x \in J^{*}$ being $\int \mu^{*}(x) d \lambda$.

These ideas can be further extended to give more general "direct products" for certain classes of $F^{\prime}$-measure algebras, but it seems to be difficult to define a satisfactory product for two arbitrary $F^{\prime}$-measure algebras. Even the product $(E, \lambda) \times E^{\prime}$ discussed above can behave in an unexpected way; for example, it can be strictly $F^{\prime}$-isomorphic to $(E, \lambda)$ (see 3.7 below).

3.3 A uniqueness theorem. It is not hard to see that a finite $F^{\prime}$-measure $\lambda$ on a Boolean $\sigma$-algebra $E$ is determined by its values on any finitely additive sub-algebra $A$ which generates $E$. We shall show that these values in fact determine $E$ too. This could be seen by a direct transfinite induction argument, but the details become rather awkward; the proof we give depends on the construction outlined in 3.1.

TheOREm 1. Let $A^{1}, A^{2}$ be finitely additive sub-algebras of two $F^{\prime}$-measure algebras $\left(E^{1}, \lambda^{1}\right),\left(E^{2}, \lambda^{2}\right)$ respectively, and suppose $\xi$ is a (finitely additive) isomorphism of $A^{1}$ onto $A^{2}$ such that $\lambda^{2}(\xi(a))=\lambda^{1}(a)$ for all $a \in A^{1}$. Suppose further that $\lambda^{1}$ is $\sigma$-finite on $A^{1}\left(^{7}\right)$. Then $\xi$ can be extended to an $F^{\prime}$-measurepreserving $\sigma$-isomorphism $\xi^{*}$ of $\mathrm{B}\left(A^{1}: E^{1}\right)$ onto $\mathrm{B}\left(A^{2}: E^{2}\right)$.

There is evidently no loss in assuming that $E^{i}=\mathbb{B}\left(A^{i}: E^{i}\right)(i=1,2)$. Further, we may assume $\lambda^{1}\left(e^{1}\right)\left(=\lambda^{2}\left(e^{2}\right)\right) \ll \infty$, since in the general case we write $e^{1}=\sup a_{n}(n=1,2, \cdots)$ where $a_{n} \in A$ and $\lambda^{1}\left(a_{n}\right) \ll \infty$, and consider the ideals $E^{1}\left(a_{n}\right)$ and $E^{2}\left(\xi\left(a_{n}\right)\right)$ separately. Let $E^{i *}$, with $F^{\prime}$-measure $\lambda^{i *}$, be the

(7) That is, $e^{\prime}$ is expressible as sup $a_{n}(n=1,2, \cdots)$ where $a_{n} \in A^{1}$ and $\lambda^{1}\left(a_{n}\right) \ll \infty$. It follows, of course, that $\lambda^{2}$ is $\sigma$-finite on $A^{2}$. 
product $\left(E^{i}, \lambda^{i}\right) \times E^{\prime}$ described in 3.1 ; we shall show that $\xi$ can in fact be extended to give a $\lambda^{*}$-preserving isomorphism between $E^{1 *}$ and $E^{2 *}$.

Let $A_{1}{ }^{*}$ denote the subset of $E^{1 *}$ consisting of all those elements $b$ which can be expressed in the form

$$
b=\sup \left(a_{n} \times y_{n}^{\prime}\right) \quad(n=1,2, \cdots),
$$

where $a_{n} \in A^{1}, y_{n}^{\prime} \in E^{\prime}$, sup $y_{n}^{\prime}=e^{\prime}$, and the elements $y_{n}^{\prime}$ are disjoint. The subset $A_{2}^{*}$ of $E^{2 *}$ is defined similarly. Clearly $A_{i}^{*}$ is a finitely additive sub-algebra of $E^{i *}$. For each $b \in A_{1}^{*}$, we express $b$ in the form (1) (in any way), and define $\xi^{\prime}(b)=\sup \left(\xi\left(a_{n}\right) \times y^{\prime}\right) \in A_{2}^{*}$; it can be verified (by an argument similar to that in $\left[4\right.$, pp. 319,320]) that $\xi^{\prime}$ is single-valued, and is a finitely additive isomorphism of $A_{1}^{*}$ onto $A_{2}^{*}$, and that $\lambda^{2 *}\left(\xi^{\prime}(b)\right)=\lambda^{1 *}(b)$ for all $b \in A_{1}^{*}$.

Next, let $C_{i}(i=1,2)$ denote the set of elements $x \in E^{i *}$ having the following property: Given $\epsilon>0$, there exists $b \in A_{i}^{*}$ such that $\lambda^{i *}(x+b) \leqq \epsilon$. (Here " + " denotes the symmetric difference.) We assert:

$$
\text { If } b_{n} \in A_{i}^{*}(n=1,2, \cdots) \text {, then } \sup b_{n} \in C_{i} .
$$

For, on replacing $b_{n}$ by sup $\left(b_{1}, b_{2}, \cdots, b_{n}\right)$, we may suppose that $b_{1} \leqq b_{2} \leqq \cdots$. Let sup $b_{n}=b$; then $\lambda^{i *}\left(b_{1}\right) \leqq \lambda^{i *}\left(b_{2}\right) \leqq \cdots \leqq \lambda^{i *}(b)$, and $\lambda^{i *}\left(b_{n}\right) \rightarrow \lambda^{i *}(b)$ as $n \rightarrow \infty$. By an "Egoroff argument" [4, p. 286], there exist disjoint elements $z_{k}^{\prime} \in E^{\prime}(k=1,2, \cdots)$ such that (i) $\sup z_{k}^{\prime}=e^{\prime}$, (ii) $\lambda^{i *}\left(b_{n}\right)$ $\geqq \lambda^{i *}(b)-\epsilon$ on $z_{k}^{\prime}$ whenever $n \geqq$ some $n_{k}$. Let $b_{n}$, expressed in the form (1), be $\sup _{m}\left(a_{n m} \times y_{n m}^{\prime}\right)$, and define $c=\sup b_{n_{k}}\left(e \times z_{k}^{\prime}\right)=\sup _{m, k}\left\{a_{n_{k} m} \times\left(y_{n_{k} m}^{\prime} z_{\mathbf{k}}^{\prime}\right)\right\}$; thus $c \in A_{i}^{*}, c \leqq b$, and $\lambda^{i *}(b-c) \leqq \epsilon$ (on $\left.e^{\prime}\right)$. This establishes (2).

It is now easy to see that $C_{i}$ is closed under complements and under countable suprema; and since $C_{i} \supset A_{i}{ }^{*}, C_{i}$ contains all rectangles $\left(a \times y^{\prime}\right)$, where $a \in A^{i}$ and $y^{\prime} \in E^{\prime}$. Hence $C_{i}$ contains the Borel field in $E^{i *}$ generated by these rectangles, and so contains all rectangles $x \times y^{\prime}$ where $x \in E^{i}$ (and $y^{\prime} \in E^{\prime}$ ), and so their Borel field. Thus finally $C_{i}=E^{i *}$.

It is now easy to extend $\xi^{\prime}$ to the whole of $E^{1 *}$ by the "Cauchy sequence" principle (cf. [4, p. 321]). Given $h \in E^{1 *}$, we have $h \in C_{1}$, and so can find elements $b_{n} \in A_{1}{ }^{*}$ such that $\lambda^{1 *}\left(h+b_{n}\right) \leqq 1 / 2^{n}$. Let $\xi^{\prime}\left(b_{n}\right)=c_{n}$; we easily verify that $\lim c_{n}$ exists (i.e., $\lim \sup c_{n}=\lim \inf c_{n}$ ), and we define $\xi^{\prime \prime}(h)=\lim c_{n}$. It can be verified that $\xi^{\prime \prime}$ is single-valued, and is a $\sigma$-isomorphism of $E^{1 *}$ onto $E^{2 *}$ extending $\xi^{\prime}$ and such that $\lambda^{2 *}\left(\xi^{\prime \prime}(h)\right)=\lambda^{1 *}(h)\left(h \in E^{1 *}\right)$. Further, $\xi^{\prime \prime}$ maps the set of rectangles $x \times e^{\prime}\left(x \in E^{1}\right)$ onto a Borel field $B$ in $E^{2 *}$ which contains the images $\xi^{\prime}\left(a \times e^{\prime}\right)\left(a \in A^{1}\right)$, i.e., the rectangles $\xi(a) \times e^{\prime}$. Hence $\mathbb{B}$ contains all the rectangles $t \times e^{\prime}\left(t \in E^{2}\right)$. A similar argument applied to $\left(\xi^{\prime \prime}\right)^{-1}$ now shows that $\mathbb{B}$ consists of all such rectangles. Thus $\xi^{\prime \prime}$ induces a mapping $\xi^{*}$ of $E^{1}$ onto $E^{2}$ by the rule $\xi^{\prime \prime}\left(x \times e^{\prime}\right)=\xi^{*}(x) \times e^{\prime}$; and clearly $\xi^{*}$ is a $\sigma$-isomorphism, agreeing with $\xi$ on $A^{1}$, and such that $\lambda^{2}\left(\xi^{*}(x)\right)=\lambda^{1}(x)$ for all $x \in E^{1}$.

3.4 We remark that, in Theorem 1 , the extension $\xi^{*}$ of $\xi$ to $\mathcal{B}\left(A^{1}: E^{1}\right)$ is in fact unique; for if $\eta$ is another isomorphism with the properties specified for 
$\xi^{*}, \eta$ and $\xi^{*}$ will agree on a $\sigma$-subalgebra of $E^{1}$ which contains $A^{1}$, and which therefore is $\mathrm{B}\left(A^{1}: E^{1}\right)$.

The following example shows that the condition that $\lambda^{1}$ be $\sigma$-finite on $A^{1}$ cannot be omitted from Theorem 1. Let $E^{1}=E^{2}=$ algebra of measurable sets modulo null sets on the unit interval, and let $A^{1}=A^{2}$ consist of all finite unions of intervals, with $\xi$ as the identity mapping of $A^{1}$ onto $A^{2}$. For $\lambda^{1}$ we take a positive $\sigma$-finite numerical measure on $E^{1}$ such that every non-null open set has infinite $\lambda^{1}$-measure. (For instance, let $\lambda^{1}(x)=\int_{x} \sum d t /\left(2^{n}\left(\rho_{n}-t\right)^{2}\right)$, where $\left\{\rho_{n}\right\}$ is an enumeration of the rational numbers.) Take $\lambda^{2}(x)=2 \lambda^{1}(x)$. The remaining conditions of Theorem 1 are satisfied (with $\lambda^{2}(\xi(a))=\infty=\lambda^{1}(a)$ for all nonzero $\left.a \in A^{1}\right)$; but the only isomorphism $\xi^{*}$ of $E^{1}$ onto $E^{2}$ which extends $\xi$ is the identity mapping, and the condition $\lambda^{2}\left(\xi^{*}(x)\right)=\lambda^{1}(x)$ is violated.

$3.5 A$ characterization of $(E, \lambda) \times E^{\prime}$. We recall that a "strict $F^{\prime}$-isomorphism" between two $F^{\prime}$-measure algebras is a $(\sigma-)$ isomorphism between them which preserves the $F^{\prime}$-measures.

Theorem 2. Let $(E, \lambda)$ be an $F^{\prime}$-measure algebra, where $F^{\prime}=F\left(E^{\prime}\right)$, and let $\left(E^{*}, \lambda^{*}\right)$ be any $F^{\prime}$-measure algebra for which (i) $E^{*}$ contains sub-algebras $E_{0}$, $E_{0}^{\prime}$, respectively $\sigma$-isomorphic to $E, E^{\prime}$, such that whenever $x_{0} \in E_{0}$ and $y_{0}^{\prime} \in E_{0}^{\prime}$ correspond to $x \in E$ and $y^{\prime} \in E^{\prime}$ we have $\lambda^{*}\left(x_{0} y_{0}^{\prime}\right)=\lambda(x) \chi\left(y^{\prime}\right)$, (ii) $E^{*}=\mathbb{B}\left(E_{0}\right.$ $\left.\cup E_{0}^{\prime}\right)$. Then $\left(E^{*}, \lambda^{*}\right)$ is strictly $F^{\prime}$-isomorphic to the product $(E, \lambda) \times E^{\prime}$ (with its standard $F^{\prime}$-measure).

As was remarked in $3.1,(E, \lambda) \times E^{\prime}$ has properties (i) and (ii). To establish Theorem 2 , we apply Theorem 1 to $\left(E^{1}, \lambda^{1}\right)=(E, \lambda) \times E^{\prime}$ and $\left(E^{2}, \lambda^{2}\right)=\left(E^{*}, \lambda^{*}\right)$. We take $A^{1}$ to consist of all "rectangle-sums" $b=\mathrm{U}\left(x_{n} \times y_{n}^{\prime}\right)(n=1,2, \cdots)$ where $x_{n} \in E, y_{n}^{\prime} \in E^{\prime}$, sup $y_{n}^{\prime}=e^{\prime}$, and the elements $y_{n}^{\prime}$ are disjoint. Similarly, $A^{2}$ consists of all elements expressible as $b_{0}=\sup x_{0 n} y_{0 n}^{\prime}$ where $x_{0 n} \in E_{0}$, $y_{0 n}^{\prime} \in E_{0}^{\prime}, \sup y_{0 n}^{\prime}=e^{*}$, and the elements $y_{0 n}^{\prime}$ are disjoint. The "natural" mapping $\xi(b)=b_{0}$ is easily seen to give a single-valued (finitely additive) isomorphism of $A^{1}$ onto $A^{2}$ such that $\lambda^{2}(\xi(b))=\lambda^{1}(b)$; and Theorem 1 applies.

3.6 It was remarked in 3.1 that $(E, \lambda) \times E^{\prime}$ provides a full-valued extension (see 2.4) of the $F^{\prime}$-integral $\phi$ (corresponding to $\lambda$ ) on $F(E)$. We now show that it is, in a sense, the smallest algebra which does so.

TheOREM 3. Let $\tilde{\phi}$ be a full-valued $F^{\prime}$-integral on $F(\tilde{E})$ which extends the $F^{\prime}$-integral $\phi$ on $F(E)$. Then $\tilde{\phi}$ is also an extension of the extension $\phi^{*}$ of $\phi$ to $F\left((E, \lambda) \times E^{\prime}\right)$, where $\lambda$ is the $F^{\prime}$-measure which corresponds to $\phi$. In particular, $(E, \lambda) \times E^{\prime}$ is isomorphic to a subalgebra of $E$.

Write $\bar{\lambda}$ for the $F^{\prime}$-measure on $E$ which corresponds to $\tilde{\phi}$; thus $\bar{\lambda}(x)$ $=\tilde{\phi}(\chi(x))(x \in E)$. By hypothesis, $E$ contains a subalgebra $E_{1}$ isomorphic to $E$; and, by $2.3(2), E$ contains a subalgebra $E_{1}^{\prime}$ isomorphic to $E^{\prime}$ such that $\tilde{\lambda}\left(x y_{1}^{\prime}\right)=\lambda(x) \chi\left(y^{\prime}\right) \quad$ whenever $\quad y_{1}^{\prime} \in E_{1}^{\prime} \quad$ corresponds to $y^{\prime} \in E^{\prime}$. Let 
$E_{1}^{*}=\mathscr{B}\left(E_{1} \cup E_{1}^{\prime} ; \widetilde{E}\right)$; then, by Theorem $2, E_{1}^{*}$ is isomorphic to $E^{*}=(E, \lambda) \times E^{\prime}$ by an isomorphism which sends $\lambda$ in to $\lambda^{*}$, the standard $F^{\prime}$-measure on $(E, \lambda)$ $\times E^{\prime}$. Since $E_{1}^{*}$ is a subalgebra of $\tilde{E}$, the whole theorem follows.

We remark further that this embedding of $E^{*}$ in $E$ is a "natural" one, in that the "rectangle" $x \times y^{\prime}$ of $E^{*}$ (where $x \in E$ and $y^{\prime} \in E^{\prime}$ ) is mapped onto the element $x_{1} y_{1}^{\prime}$ of $\tilde{E}$.

3.7 Corollary. If $\phi$ is a full-valued $F^{\prime}$-integral on $F(E)$, then $(E, \lambda) \times E^{\prime}$ is isomorphic to $E$.

For Theorem 3, applied to $\tilde{E}=E, \tilde{\phi}=\phi$, gives that $\left(E^{*}, \lambda^{*}\right)=(E, \lambda) \times E^{\prime}$ is isomorphic to a subalgebra of $E$. The "rectangle" $x \times e^{\prime}$ of $E^{*}$ must correspond here to the element $x e(=x)$ of $E$, so the image of $E^{*}$ is the whole of $E$. In fact, $E^{\prime}$ is now, to within isomorphism, a subalgebra of $E$; and the isomorphism between $E^{*}$ and $E$ makes $x \times y^{\prime}\left(x \in E, y^{\prime} \in E^{\prime}\right)$ correspond to the element $x y^{\prime}$ of $E$.

\section{Kernel representation for relaxed $F^{\prime}$-integrals.}

4.1 Strict forms. Let $\phi$ be a relaxed $F^{\prime}$-integral (cf. 2.2) on $F(E)$, where $F^{\prime}=F\left(E^{\prime}\right)$. Let $E_{1}^{\prime}$ be the principal ideal $E^{\prime}[\phi(1)]$ of $E^{\prime}$, and let $E_{1}$ be the principal ideal $E(e-\sup \{b \mid \lambda(b)=0\})$ of $E$, where $\lambda$ is the relaxed $F^{\prime}$-measure corresponding to $\phi$ (i.e., $\lambda(x)=\phi(\chi(x)))$. Write $F_{1}=F\left(E_{1}\right), F_{1}^{\prime}=F\left(E_{1}^{\prime}\right)$. Then a (strict) $F^{\prime}$-integral $\phi_{1}$ can be defined on $F_{1}$ as follows. $F_{1}$ can be regarded as imbedded in $F$, the function $f_{1} \in F_{1}$ corresponding to the function $f \in F$ which agrees with $f_{1}$ on $e_{1}$ and is 0 on $e-e_{1}$. Further, $\phi(f)$ will vanish on $e^{\prime}-[\phi(1)]$; when restricted to $[\phi(1)]$, it gives a function $f_{1}^{\prime} \in F_{1}^{\prime}$. We define $\phi_{1}\left(f_{1}\right)=f_{1}^{\prime}$ (for $f_{1} \in F_{1}^{+}$), and readily verify that $\phi_{1}$ satisfies all the conditions $(2.2, \alpha-\delta)$ for a (strict) $F_{1}^{\prime}$-integral on $F_{1}$. We call this $\phi_{1}$ the "strict form" of $\phi$. Similarly a (strict) $F_{1}^{\prime}$-measure $\lambda_{1}$, called the strict form of $\lambda$, is defined on $E_{1}$ by the rule: for each $x \in E_{1}, \lambda_{1}(x)=\lambda(x)$ restricted to $e_{1}^{\prime}$. It is easy to see that $\lambda_{1}$ and $\phi_{1}$ correspond to each other-i.e., $\lambda_{1}(x)=\phi_{1}(\chi(x)) \quad\left(x \in E_{1}\right)$, $\phi_{1}(f)=\int f d \lambda_{1}\left(f \in F_{1}^{+}\right)$. When $\phi_{1}$ (or $\left.\lambda_{1}\right)$ is known, $\phi$ is completely determined, since if $f \in F^{4}, \phi(f)=\phi_{1}\left(f\right.$ restricted to $\left.e_{1}\right)$ on $e_{1}^{\prime}, 0$ on $e^{\prime}-e_{1}^{\prime}$. Thus, by applying the theory of $F^{\prime}$-measures and integrals to $\phi_{1}$ and $\lambda_{1}$, the corresponding properties of $\phi$ and $\lambda$ can be read off.

A relaxed $F^{\prime}$-integral $\phi$ is called full-valued if it satisfies 2.3 (1). It is easy to see that $\phi$ is full-valued if and only if its strict form, $\phi_{1}$, is full-valued. (But 2.3 (2) does not apply unchanged to relaxed full-valued $F^{\prime}$-integrals; $E$ and $E^{\prime}$ must be replaced by the subalgebras $E_{1}$ and $E_{1}^{\prime}$.)

The construction of $(E, \lambda) \times E^{\prime}$ (3.1) applies unchanged when $\lambda$ is a relaxed $F^{\prime}$-measure; but it is easy to see that the presence of extra null sets results in $(E, \lambda) \times E^{\prime}$ being isomorphic to $\left(E_{1}, \lambda_{1}\right) \times E_{1}^{\prime}, \lambda_{1}$ being the strict form of $\lambda$.

We have:

TheOREM 4. Let $\phi$ be a relaxed $F^{\prime}$-integral on $F=F(E)$. Then $\phi$ has a minimal full-valued extension, $\phi^{*}$. 
That is, there exists a relaxed $F^{\prime}$-integral $\phi^{*}$ on $F^{*}=F\left(E^{*}\right)$, where $E^{*}$ is a $\sigma$-algebra containing $E$ as a subalgebra, such that (i) $\phi^{*}(f)=\phi(f)$ whenever $f \in F^{+}$(regarded as imbedded in $F^{*}$ in the natural way), (ii) if $\tilde{E}$ is any other algebra having $E$ as a subalgebra, and if $\widetilde{\phi}$ is any relaxed $F^{\prime}$-integral on $F(\widetilde{E})$ which agrees with $\phi$ on $F$, then (after an isomorphism of $\tilde{E}$ which leaves each element of $E$ invariant) $E^{*}$ is a subalgebra of $\widetilde{E}$, and $\widetilde{\phi}$ agrees with $\phi^{*}$ on $F^{*}$.

To construct $E^{*}$ and $\phi^{*}$, let $\lambda_{1}$ be the strict form of the relaxed $F^{\prime}$-measure $\lambda$ corresponding to $\phi$, and define $E^{*}$ to be the direct sum of $\left(E_{1}, \lambda_{1}\right) \times E_{1}^{\prime}$ and $E\left(e-e_{1}\right)$. To within an isomorphism, $E$ is a subalgebra of $E^{*}$, the element $x$ of $E$ corresponding to $\left(x e_{1} \times e_{1}^{\prime}\right) \bigvee\left(x-e_{1}\right)$ in $E^{*}$. Each $f \in F\left(E^{*}\right)^{+}$can be written uniquely as $f_{1}+f_{2}$, where $f_{1}$ is 0 on $e-e_{1}$ and $f_{2}$ is 0 on $e_{1} \times e_{1}^{\prime}$. We define $\phi^{*}(f)=\phi_{1}^{*}\left(f_{1}\right), \phi_{1}^{*}$ being the standard (full-valued) extension of $\phi_{1}$ to $F\left(\left(E_{1}, \lambda_{1}\right) \times E_{1}^{\prime}\right)$. It is easy to see that $\phi^{*}$ is a full-valued relaxed $F^{\prime}$-integral on $F\left(E^{*}\right)$ (in fact, the strict form of $\phi^{*}$ is $\phi_{1}^{*}$ ), and that $\phi^{*}$ extends $\phi$. Finally, the minimality of this extension (property (ii) above) follows easily from Theorem 3 (3.6).

4.2 Kernel representation. Let $\phi, \psi$ be relaxed $F^{\prime}$-integrals on $F=F(E)$, where $F^{\prime}=F\left(E^{\prime}\right)$. If, for some fixed finite $k \in F^{+}$, we have

$$
\psi(f)=\phi(k f) \text { for all } f \in F^{+},
$$

we say that $\psi$ has a kernel representation in terms of $\phi$, with kernel $k$. It is easy to see that (1) will then hold, even if $f \notin F^{+}$, provided either side has a meaning. Further, it is enough that (1) should hold whenever $f$ is a characteristic function.

We shall later need the following lemma, which shows that the requirement that $k$ be finite is not really a restriction.

Lemma. Suppose $E$ is a subalgebra of $E^{*}, \phi^{*}$ is a strict $F^{\prime}$-integral on $F^{*}=F\left(E^{*}\right)$, and $\psi$ is a relaxed $F^{\prime}$-integral on $F=F(E)$. If for some $k \in F^{*+}$ we have $\psi(f)=\phi^{*}(k f)$ (for all $f \in F^{+}$), then $k$ is finite, and $\psi$ can be extended to a relaxed $F^{\prime}$-integral $\psi^{*}$ on $F^{*}$ which has a kernel representation in terms of $\phi^{*}$.

For let $z=$ set in $E^{*}$ where $k$ is $\infty$. Since $\psi$ is $\sigma$-finite, there exist elements $a_{n} \in E$ such that $\sup a_{n}=e=e^{*}$ and $\psi\left(\chi\left(a_{n}\right)\right) \ll \infty$. Now, if $\lambda^{*}$ denotes the (strict) $F^{\prime}$-measure corresponding to $\phi^{*}$, we have $\infty \lambda^{*}\left(z a_{n}\right)=\phi^{*}\left(\infty \chi(z) \chi\left(a_{n}\right)\right)$ $\leqq \phi^{*}\left(k \chi\left(a_{n}\right)\right)=\psi\left(\chi\left(a_{n}\right)\right) \ll \infty$, and so $\lambda^{*}\left(z a_{n}\right)=0$. Thus $z a_{n}=o(n=1,2, \cdots)$, and therefore $z=o$. This proves that $k$ is finite. To extend $\psi$, we merely define $\psi^{*}\left(f^{*}\right)=\phi^{*}\left(k f^{*}\right)\left(f^{*} \in F^{*+}\right)$.

4.3 If $\psi$ has a kernel representation in terms of $\phi$, then we have $[\psi(f)]$ $\leqq[\phi(\infty f)]=[\infty \phi(f)]$, and so $[\psi(f)] \leqq[\phi(f)]$ for all $f \in F^{+}$. The converse holds, as we now show, provided that $\phi$ is full-valued. We give later (4.4) an example to show that the converse may be false without this proviso.

TheOREM 5. Let $\phi, \psi$ be relaxed $F^{\prime}$-integrals on $F=F(E)$, such that $[\psi(f)]$ $\leqq[\phi(f)]$ for all $f \in F^{+}$. Suppose $\phi$ is full-valued. Then $\psi$ is also full-valued, and $\psi$ has a kernel representation in terms of $\phi$. 
REMARK. In the numerical case, in which $F^{\prime}$ consists of the functions on a single atom, $\phi$ is automatically full-valued, and Theorem 5 then becomes the Radon-Nikodym theorem (see, for example, [7, pp. 137-139]).

Proof of Theorem 5. Let $\lambda, \mu$ be the relaxed $F^{\prime}$-measures corresponding to $\phi, \psi$ respectively, and let $\phi_{1}, \lambda_{1}$ be the strict forms of $\phi, \lambda$, so that $\lambda_{1}$ is an $F\left(E_{1}^{\prime}\right)$-measure on $E_{1}$, in the notation of 4.1. Here $E_{1}^{\prime}=E^{\prime}\left(e_{1}^{\prime}\right)$ where $e_{1}^{\prime}=[\phi(1)]=[\lambda(e)]=\left[\lambda\left(e_{1}\right)\right]$. Define $\mu_{1}(x)=\mu(x)$ restricted to $e_{1}^{\prime}$. Then $\mu_{1}$ is a relaxed $F\left(E_{1}^{\prime}\right)$-measure on $E_{1}$, and $\mu(x)$ is the function on $e^{\prime}$ which agrees with $\mu_{1}(x)$ on $e_{1}^{\prime}$ and is 0 on $e^{\prime}-e_{1}^{\prime}$ (for $\left.[\mu(x)] \leqq[\lambda(x)]\right)$. Further, $\left[\mu_{1}(x)\right]$ $\leqq\left[\lambda_{1}(x)\right]$ when $x \in E_{1}$, and $\mu_{1}(x)=0$ whenever $x \leqq e-e_{1}$ (for then $\left.[\lambda(x)]=o\right)$. We shall show that there exists a finite non-negative $k_{1} \in F\left(E_{1}^{\prime}\right)$ such that

$$
\mu_{1}(x)=\phi_{1}\left(k_{1} \chi(x)\right) \text { whenever } x \in E_{1} .
$$

It will then follow that $\mu(x)=\phi(k \chi(x))$ whenever $x \in E, k$ being an arbitrary extension of $k_{1}$ to $e$, and the desired kernel relation

$$
\psi(f)=\phi(k f)
$$

will follow in turn (cf. [6, 2.3]). The full-valuedness of $\psi$ is then an easy consequence of (2) and the fact that $\phi$ is full-valued. Thus it suffices to prove (1); and, in doing so, we may simplify the notation by omitting the suffixes 1 . That is, we may assume in what follows that $\phi$ is a strict $F^{\prime}$-integral on $F$ (so that $\lambda$ is a strict $F^{\prime}$-measure on $E$ ). We have, by assumption,

$$
[\mu(x)] \leqq[\lambda(x)]
$$

$$
(x \in E) \text {. }
$$

Since $\phi$ is full-valued, we can (cf. 2.3 (2)) identify $E^{\prime}$ with a sub-algebra of $E$, and then have

$$
\lambda\left(x y^{\prime}\right)=\lambda(x) \chi\left(y^{\prime}\right) \quad\left(x \in E, y \in E^{\prime} \subset E\right) .
$$

We shall deduce:

$$
\mu\left(x y^{\prime}\right)=\mu(x) \chi\left(y^{\prime}\right) \quad\left(x \in E, y^{\prime} \in E^{\prime}\right) .
$$

In fact, $\left[\mu\left(x y^{\prime}\right)\right] \leqq\left[\lambda\left(x y^{\prime}\right)\right] \leqq y^{\prime}$, from (4), and therefore $\mu\left(x y^{\prime}\right)=\mu\left(x y^{\prime}\right) \chi\left(y^{\prime}\right)$. Similarly $\mu\left(x-y^{\prime}\right)=\mu\left(x-y^{\prime}\right) \chi\left(e-y^{\prime}\right)$. Hence

$$
\mu(x) \chi\left(y^{\prime}\right)=\left\{\mu\left(x y^{\prime}\right)+\mu\left(x-y^{\prime}\right)\right\} \chi\left(y^{\prime}\right)=\mu\left(x y^{\prime}\right)+0,
$$

proving (5).

Another property which will be useful is

$$
x \leqq[\lambda(x)]
$$$$
(x \in E) \text {. }
$$

To see this, apply (4) with $y^{\prime}=e-[\lambda(x)]\left(\in E^{\prime}\right)$; this gives $\lambda\left(x y^{\prime}\right)=0$, and consequently $x y^{\prime}=0$, i.e., $x \leqq[\lambda(x)]\left({ }^{8}\right)$.

(8) In fact it is not hard to see that $[\lambda(x)]=$ smallest element of $E^{\prime}$ containing $x$. 
Now, using 2.2(b), we write $e=\sup e^{n}(n=1,2, \cdots)$, where the elements $e^{n}$ are disjoint and $\mu\left(e^{n}\right), \lambda\left(e^{n}\right)$ are both finite. For each rational number $\rho$, let $Z^{n}(\rho)$ denote the set of all those elements $z$ of $E$ which have the following property:

$z \leqq e^{n}$, and whenever $t \leqq z$ we have $\mu(t) \leqq \rho \lambda(t)$. Clearly if $z \in Z^{n}(\rho)$, then so do all elements $u \leqq z$. Write $x^{n}(\rho)=\sup \left\{z \mid z \in Z^{n}(\rho)\right\}$. Then (from the countable chain condition) we have $x^{n}(\rho)=\sup z_{m}(m=1,2, \cdots)$ for a suitable sequence of elements $z_{m} \in Z^{n}(\rho)$; and, on replacing $z_{m}$ by $z_{m}-\sup \left(z_{1}, z_{2}, \cdots\right.$, $\left.z_{m-1}\right)$, we may further suppose the elements $z_{m}$ to be disjoint. If $t \leqq x^{n}(\rho)$, we have $t=\sup \left(t z_{m}\right)$ and so $\mu(t)=\sum \mu\left(t z_{m}\right) \leqq \rho \sum \lambda\left(t z_{m}\right)=\rho \lambda(t)$. This proves that $x^{n}(\rho) \in Z^{n}(\rho)$; i.e.,

$$
x^{n}(\rho) \leqq e^{n}, \text { and } \mu(x) \leqq \rho \lambda(x) \text { whenever } x \leqq x^{n}(\rho) .
$$

We next prove:

$$
\mu(y) \gg \rho \lambda(y) \text { on }[\lambda(y)] \text { whenever } y \leqq e^{n}-x^{n}(\rho) .
$$

In proving (8), we may assume $\rho \geqq 0$ and $y \neq 0$. Now consider a maximal disjoint collection (necessarily countable) of nonzero elements $y_{\alpha}$ such that (i) $y_{\alpha} \leqq y$, (ii) $\mu\left(y_{\alpha}\right) \gg \rho \lambda\left(y_{\alpha}\right)$ on $\left[\lambda\left(y_{\alpha}\right)\right]$. We first show that sup $y_{\alpha}=y$. If not, consider the nonzero element $y-\sup y_{\alpha}$; it is not in $Z^{n}(\rho)$ (being disjoint from $\left.x^{n}(\rho)\right)$, and so there is some nonzero $t \leqq y-\sup y_{\alpha}$ which fails to satisfy $\mu(t) \leqq \rho \lambda(t)$. Hence for some nonzero $y^{\prime} \in E^{\prime}$ we have $\mu\left(t y^{\prime}\right) \gg \rho \lambda\left(t y^{\prime}\right)$ on $y^{\prime}$. From (3) it follows that $\lambda\left(t y^{\prime}\right) \gg 0$ on $y^{\prime}$, and consequently (in view of (4)) $\left[\lambda\left(t y^{\prime}\right)\right]=y^{\prime}$. Thus the nonzero element $t y^{\prime}$, which is disjoint from every member of the collection $\left\{y_{\alpha}\right\}$, could have been adjoined to it, contradicting the maximality of the collection.

Thus sup $y_{\alpha}=y$. Now $\mu\left(y_{\alpha}\right) \geqq \rho \lambda\left(y_{\alpha}\right)$ (everywhere), since on $e-\left[\lambda\left(y_{\alpha}\right)\right]$ both functions are 0 . Hence $\sum \mu\left(y_{\alpha}\right) \geqq \rho \sum \lambda\left(y_{\alpha}\right)$; that is, $\mu(y) \geqq \rho \lambda(y)$. Further, at each point in the union of the sets $\left[\lambda\left(y_{\alpha}\right)\right]$ (in a realization of $E$ ) there is some term $\mu\left(y_{\alpha}\right)$ which is strictly greater than the corresponding term $\lambda\left(y_{\alpha}\right)$. Since $\sum \mu\left(y_{\alpha}\right)$ is finite (for $\left.y \leqq e^{n}\right)$, we have $\mu(y) \gg \rho \lambda(y)$ on sup $\left[\lambda\left(y_{\alpha}\right)\right]$, i.e., on $[\lambda(y)]$, and $(8)$ is established.

From (7) and (8) it is easy to deduce that, for any rational numbers $\rho, \sigma$,

$$
\begin{aligned}
x^{n}(\rho) & \leqq x^{n}(\sigma) \text { if } \rho \leqq \sigma ; \\
\inf \left\{x^{n}(\rho) \mid \rho>\sigma\right\} & =x^{n}(\sigma) .
\end{aligned}
$$

It follows that if we define, for each point $s$ of $e^{n}$ (in a realization of $E$ )

$$
k_{n}(s)=\inf \left\{\rho \mid s \in x^{n}(\rho)\right\},
$$

then the resulting function $k_{n}$ satisfies

$$
k_{n} \in F\left(E\left(e^{n}\right)\right), \quad \text { and } \quad x^{n}(\rho) \text { is the set where } k_{n} \leqq \rho .
$$

Now clearly $x^{n}(\rho)=0$ if $\rho<0$ (from (7)); hence $k_{n} \geqq 0$. Again, let 
$y=e^{n}-\sup _{\rho} x^{n}(\rho)$; then (from (8)) $\mu(y) \gg \rho \lambda(y)$ on $[\lambda(y)]$ for every rational number $\rho$; but $\mu(y)$ is finite (for $y \leqq e^{n}$ ), and therefore $\lambda(y)=0$, i.e., $y=0$. Thus $\sup _{\rho} x^{n}(\rho)=e^{n}$, and we have proved

$$
0 \leqq k_{n} \ll \infty \quad\left(\text { on } e^{n}\right) .
$$

Let $k_{n}^{*}$ be the element of $F$ which agrees with $k_{n}$ on $e^{n}$ and is 0 on $e-e^{n}$, and write $k=\sum k_{n}^{*}$. Since the elements $e^{n}$ are disjoint, we have

$$
k \in F, \text { and } 0 \leqq k \ll \infty \quad(\text { on } e \text { ). }
$$

To complete the proof of (1), it will now suffice to prove

$$
\mu(z)=\phi\left(k_{n}^{*} \chi(z)\right) \quad \text { whenever } z \leqq e^{n} .
$$

For we then have, for any $x \in E, \mu\left(x e^{n}\right)=\phi\left(k_{n}^{*} \chi\left(x e^{n}\right)\right)=\phi\left(k \chi\left(x e^{n}\right)\right)$; and summation with respect to $n$ gives $\mu(x)=\phi(k \chi(x))$.

Let $m$ be a positive integer, fixed for the present, and write $y_{0}=x^{n}(0) z$, $y_{p}=\left\{x^{n}(p / m)-x^{n}((p-1) / m)\right\} z\left(p=1,2, \cdots, z \in E\left(e^{n}\right)\right)$. Thus the elements $y_{p}(p=0,1,2, \cdots)$ are disjoint and (since sup $\left.x^{n}(\rho)=e^{n}\right)(9)$ shows that sup $y_{p}=z$. Further, (11) gives $((p-1) / m) \chi\left(y_{p}\right) \leqq k_{n}^{*} \chi\left(y_{p}\right) \leqq(p / m) \chi\left(y_{p}\right)$, whence (applying $\phi)((p-1) / m) \lambda\left(y_{p}\right) \leqq \phi\left(k_{n}^{*} \chi\left(y_{p}\right)\right) \leqq(p / m) \lambda\left(y_{p}\right)$. Again, (7) and (8) give $((p-1) / m) \lambda\left(y_{p}\right) \leqq \mu\left(y_{p}\right) \leqq(p / m) \lambda\left(y_{p}\right)$. Hence $\left(-(1 / m) \lambda\left(y_{p}\right)\right.$ $\leqq \mu\left(y_{p}\right)-\phi\left(k_{n}^{*} \chi\left(y_{p}\right)\right) \leqq(1 / m) \lambda\left(y_{p}\right)$. Summing over $p$ gives

$$
-(1 / m) \lambda(z) \leqq \mu(z)-\phi\left(k_{n}^{*} \chi(z)\right) \leqq(1 / m) \lambda(z) .
$$

This is true for every positive integer $m$, and $\lambda(z)$ is finite; thus (14) follows, and the theorem is proved.

4.4 An example. The following example shows that Theorem 5 would not be true if the condition that $\phi$ be full-valued were omitted. Let $E$ be the algebra of measurable sets modulo null sets on the unit interval $0 \leqq y \leqq 1$, and let $E^{\prime}$ be the similar algebra for the interval $0 \leqq x \leqq 1$; we define two $F\left(E^{\prime}\right)$ integrals $\phi, \psi$ on $F(E)$ as follows. There exist (see [1]) two complementary measurable sets $H, K$ in the unit square $0 \leqq x, y \leqq 1$, each of which meets every product set $A \times B$ (where $A, B$ are non-null measurable subsets of the unit intervals on the respective axes) in a set of positive measure. We define $\phi(f)=g$ where $g(x)=\int_{0}^{1} f(y) \chi_{H}(x, y) d y, \quad \psi(f)=g^{\prime}, g^{\prime}(x)=\int_{0}^{1} f(y) \chi_{K}(x, y) d y$. This amounts to defining the corresponding $F^{\prime}$-measure $\lambda$ on $E$ by: $\lambda(z)$ $=$ the function on $E^{\prime}$ whose value at $\alpha$ is the linear measure of the intersection $(\alpha \times z) \cap H$; and similarly for $\mu$. An application of Fubini's theorem shows that $[\lambda(z)]=e^{\prime}=[\mu(z)]$ unless $z=0$. Hence, if $f \in F(E)^{+}$, we have $[\phi(f)]=e^{\prime}=[\psi(f)]$, unless $f=0$, when $[\phi(f)]=o=[\psi(f)]$. Thus the other conditions of Theorem 5 are all satisfied, and $\phi, \psi$ are even strict $F^{\prime}$-integrals. But it is not hard to see that $\psi$ cannot have a kernel representation in terms of $\phi$. We omit the proof, since a stronger statement will be proved later (5.7): 
there are no extensions $\psi^{*}, \phi^{*}$ of $\psi$ and $\phi$ to any larger function space for which $\psi^{*}$ can have a kernel representation in terms of $\phi^{*}$. In particular, there is no measurable function $k$ on the unit square for which $\psi$ can be expressed in the form $\psi(f)=g^{\prime}$ where $g^{\prime}(x)=\int_{0}^{1} k(x, y) \chi_{H}(x, y) f(y) d y$.

4.5 An illustration. Finally we mention a simple example to which Theorem 5 does apply. Let $E$ be the algebra of measurable sets modulo null sets on the unit square $0 \leqq x, y \leqq 1$, and let $E^{\prime}$ consist of the measurable sets modulo null sets on $0 \leqq x \leqq 1$. The $F^{\prime}$-integral $\phi$ given by: $\phi(f)=f^{\prime}$ where $f^{\prime}(x)$ $=\int_{0}^{1} f(x, y) d y$ is full-valued. Moreover, if $f \in F^{+},[\phi(f)]=$ set of $x$ 's for which $f(x, y)>0$ for a set of $y$ 's of positive measure. Hence the theorem: Let $f \rightarrow g^{\prime}$ be a mapping of $\mathrm{F}^{+}$in $\mathrm{F}^{\prime+}$ which is countably additive and $\sigma$-finite (in the sense of 2.2, $\alpha$ and $\beta$ ), and satisfies the condition that $g^{\prime}(x)=0$ for almost all $x$ such that $f(x, y)=0$ for almost all $y$. Then there exists a fixed finite nonnegative measurable function $k(x, y)$ such that

$$
g^{\prime}(x)=\int_{0}^{1} k(x, y) f(x, y) d y \quad \text { (for all measurable non-negative } f \text { ). }
$$

The conditions imposed on the mapping $f \rightarrow g^{\prime}$ are obviously necessary as well as sufficient.

\section{Dominance and extended kernel representation.}

5.1 The previous theorem gives sufficient conditions for the kernel representation of $\psi$ in terms of $\phi$, in the original function space $F$. In the present section, we investigate the corresponding question for suitable extensions of $\psi$ and $\phi$, where the kernel can be drawn from a larger function space. (This is, in fact, the usual situation in applications.) The answer to the question (Theorem 7 and corollary, 5.4 and 5.5) will be obtained by combining Theorem 5 with the following simple result:

Theorem 6. Let $\mu$ be a relaxed $F^{\prime}$-measure on an $F^{\prime}$-measure algebra $(E, \lambda)$, such that $\mu(x) \leqq \lambda(x)$ for all $x \in E$. Then there exists a unique extension $\mu^{*}$ of $\mu$ to a relaxed $F^{\prime}$-measure on $\left(E^{*}, \lambda^{*}\right)=(E, \lambda) \times E^{\prime}$ such that $\mu^{*}(z) \leqq \lambda^{*}(z)$ for all $z \in E^{*}$.

We regard $E$ as imbedded in $E^{*}$ in the natural way; thus the statement that $\mu^{*}$ is an extension of $\mu$ means that $\mu^{*}\left(x \times e^{\prime}\right)=\mu(x)$ for all $x \in E$.

Proof. We take $E$ and $E^{\prime}$ to be realized by the open-closed subsets (modulo sets of first category) of their representation spaces $R, S^{\prime}$, and recall (cf. 3.1) that $E^{*}$ is then realized by the Borel field $\mathcal{B}$ generated by the rectangles $r=x \times y^{\prime}\left(x \in E, y^{\prime} \in E^{\prime}\right)$ in $R \times S^{\prime}$, modulo the $\sigma$-ideal $\mathcal{N}$ of those sets for which $\lambda^{*}=0$. For each such rectangle we define $\mu^{*}(r)=\mu(x) \chi\left(y^{\prime}\right)$; since $\lambda^{*}(r)=\lambda(x) \chi\left(y^{\prime}\right)$, we have $\mu^{*}(r) \leqq \lambda^{*}(r)$. Now exactly the same construction as for $\lambda^{*}[6$, pp. 170,171$]$ extends $\mu^{*}$ to an "outer $F^{\prime}$-measure" on $R \times S^{\prime}$ such that $\mu^{*} \leqq \lambda^{*}$, and proves that all rectangles are $\mu^{*}$-measurable. Hence all sets in $\mathcal{B}$ are $\mu^{*}$-measurable, and all sets which are null for $\lambda^{*}$ are null for $\mu^{*}$ also, 
which means that $\mu^{*}$ gives an $F^{\prime}$-measure on $B \bmod \mathcal{N}$ having the desired properties.

To see that these properties determine $\mu^{*}$ uniquely, we note that if $\tilde{\mu}$ is any other extension of $\mu$ to $E^{*}$ such that $\tilde{\mu}(z) \leqq \lambda^{*}(z)$ (for all $z \in E$ ), then for every rectangle we have $\tilde{\mu}\left(x \times y^{\prime}\right) \leqq \lambda^{*}\left(x \times y^{\prime}\right)=\lambda(x) \chi\left(y^{\prime}\right)$. From this it follows, by the same reasoning as that proving $4.3(5)$, that $\tilde{\mu}\left(x \times y^{\prime}\right)$ $=\tilde{\mu}\left(x \times e^{\prime}\right) \chi\left(y^{\prime}\right)=\mu(x) \chi\left(y^{\prime}\right)$. Thus $\tilde{\mu}$ agrees with $\mu^{*}$ on all rectangles, and so (Theorem $1,3.3$ ) it agrees with $\mu^{*}$ on all of $E^{*}$.

We remark that essentially the same argument proves that the product $(E, \mu) \times E^{\prime}$ is algebraically isomorphic, in a natural way, to a principal ideal in $(E, \lambda) \times E^{\prime}$. This observation will be generalized later (Theorem 7, 5.4).

5.2 Corollary. Let $\phi$ be an $F^{\prime}$-integral on $F=F(E)$, with corresponding $F^{\prime}$-measure $\lambda$, and let $\psi$ be a relaxed $F^{\prime}$-integral on $F$ such that $\psi(f) \leqq \phi(f)$ whenever $f \in F^{+}$. Then there exists $k \in F^{*}=F\left\{(E, \lambda) \times E^{\prime}\right\}$ such that $0 \leqq k \leqq 1$ and $\psi(f)=\phi^{*}(k f)$ (for all $\left.f \in F^{+}\right)$, where $\phi^{*}$ denotes the standard $F^{\prime}$-integral on $F^{*}$.

For, by Theorem 6 , we can extend $\psi$ to a relaxed $F^{\prime}$-integral $\psi^{*}$ on $F^{*}$ such that $\psi^{*}(f) \leqq \phi^{*}(f)\left(f \in F^{*+}\right)$. It follows that $\left[\psi^{*}(f)\right] \leqq\left[\phi^{*}(f)\right]$; hence, since $\phi^{*}$ is full-valued, Theorem 5 gives the existence of a finite $k \in F^{*+}$ such that $\psi^{*}(f)=\phi^{*}(k f)$ whenever $f \in F^{*+}$. Finally, $k \leqq 1$; for otherwise there exist a constant $\rho>1$ and a non-null $z \in E^{*}$ such that $k \geqq \rho \chi(z)$, and there exists $t \leqq z$ such that $0<\lambda^{*}(t) \ll \infty$. But then $\lambda^{*}(t)=\phi^{*}(\chi(t)) \geqq \psi^{*}(\chi(t))=\phi^{*}(k \chi(t))$ $\geqq \rho \lambda^{*}(t)$, giving a contradiction.

REMARK. It is not hard to deduce (cf. the proof of 5.5 (4) below) the following extension of Theorem 6 . If $\phi, \psi$ are arbitrary relaxed $F^{\prime}$-integrals on $F$ such that $\psi(f) \leqq \phi(f)$ (for all $f \in F^{*}$ ), and if $\phi^{*}$ is any extension of $\phi$, say to $F^{*}$, then $\psi$ can be extended to $F^{*}$ in such a way that $\psi^{*}(f) \leqq \phi^{*}(f)\left(f \in F^{*+}\right)$.

5.3 Definition. For any relaxed $F^{\prime}$-integrals $\phi, \psi$ on $F=F(E)$, we say that $\phi$ dominates $\psi$ if there exists a sequence of relaxed $F^{\prime}$-integrals $\psi_{n}$ on $F$, and a sequence of (finite) positive constants $\rho_{n}$, such that, whenever $f \in F^{+}$,

$$
\psi(f)=\sum \psi_{n}(f), \quad \text { and } \quad \psi_{n}(f) \leqq \rho_{n} \phi(f) \quad(n=1,2, \cdots) .
$$

If $\psi$ has a kernel representation in terms of $\phi$, then certainly $\phi$ dominates $\psi$. For we then have $\psi(f)=\phi(k f)$ (for all $f \in F^{+}$) where $k$ is a fixed finite element of $F^{+}$. Let $a_{n}$ be the set (in a realization of $E$ ) where $n-1 \leqq k<n$ $(n=1,2, \cdots)$, and write $\lambda$ and $\mu$ for the relaxed $F^{\prime}$-measures which correspond to $\phi$ and $\psi$, respectively; then we clearly have

$$
\text { sup } a_{n}=e, \text { and } \mu(x) \leqq \rho_{n} \lambda(x) \quad \text { for all } x \leqq a_{n} \text {, }
$$

with $\rho_{n}=n$, and from this (1) follows easily. However, (2) is in general a stronger requirement than (1) (see 5.7(a) below), though it follows from the lemma below that (2) is equivalent to (1) if $\phi$ is full-valued.

Again, if (1) holds, then clearly 


$$
[\psi(f)] \leqq[\phi(f)],
$$

though again the converse implication is false in general (see 5.7(c)).

Lemma. If $\phi$ is full-valued, then the following statements are equivalent:

(a) $\phi$ dominates $\psi$;

(b) $[\psi(f)] \leqq[\phi(f)]$ whenever $f \in F(E)^{+}$;

(c) $\psi$ has a kernel representation in terms of $\phi$.

In fact, the implications (c) $\rightarrow$ (a) $\rightarrow$ (b) are trivial, as was observed above; that (b) implies (c) follows from Theorem 5 (4.3).

REMARK. The meaning of condition (1) above is not changed if we require that all the constants $\rho_{n}$ are to be 1 . For, if (1) holds, we may suppose (increasing $\rho_{n}$, if necessary) that each $\rho_{n}$ is a positive integer, and we have only to replace each term $\psi_{n}$ by $\rho_{n}$ terms each equal to $\left(1 / \rho_{n}\right) \psi_{n}$.

5.4 TheOREM 7. Let $\phi$ and $\psi$ be relaxed $F^{\prime}$-integrals on $F=F(E)$, where $F^{\prime}=F\left(E^{\prime}\right)$, and let $\lambda$ and $\mu$ be the corresponding relaxed $F^{\prime}$-measures. Then each of the following statements implies the others:

(1) $\phi$ dominates $\psi$;

(2) $(E, \mu) \times E^{\prime}$ is (algebraically) isomorphic to a principal ideal, say of $z$, in $(E, \lambda) \times E^{\prime}$, in such a way that for all $x \in E$ and $y^{\prime} \in E^{\prime}$ the element $x \times y^{\prime}$ (possibly null) of $(E, \mu) \times E^{\prime}$ corresponds to the element $z\left(x \times y^{\prime}\right)$ of $(E, \lambda) \times E^{\prime}$.

(3) If $\phi^{*}$ is the minimal full-valued extension of $\phi$ (Theorem 4, 4.1), say to $F^{*}$, then there is a finite $k \in F^{*+}$ such that $\psi(f)=\phi^{*}(k f)$ for all $f \in F^{+}$(and so for all $f \in F$ for which either side has a meaning).

Proof. (3) $\rightarrow(1)$. We can extend $\psi$ to $F^{*}$ by defining $\psi^{*}(f)=\phi^{*}(k f)\left(f \in F^{*+}\right)$; $\psi^{*}$ has a kernel representation in terms of $\phi^{*}$, and so (from 5.3) $\phi^{*}$ dominates $\psi^{*}$, from which it follows trivially that $\phi$ dominates $\psi$.

$(1) \rightarrow(3)$. Both $\phi$ and $\psi$ vanish on $F\left(E\left(e-e_{1}\right)\right)$, where $E\left(e_{1}\right)$ is the principal ideal on which the strict form $\lambda_{1}$ of $\lambda$ operates; hence we may restrict attention to $F\left(E\left(e_{1}\right)\right)$, and so may assume that $\phi$ is strict. Thus $F^{*}=F\left(E^{*}\right)$ where $E^{*}=(E, \lambda) \times E^{\prime}$. Now, by the remark at the end of 5.3 , we have $\psi(f)$ $=\sum \psi_{n}(f)$ (for all $f \in F^{+}$), where $\psi_{n}$ is a relaxed $F^{\prime}$-integral on $F$ and $\psi_{n}(f)$ $\leqq \phi(f)$. By Theorem 6, Corollary (5.2), there exists $k_{n} \in F^{*}$ such that $0 \leqq k_{n} \leqq 1$ and $\psi_{n}(f)=\phi^{*}\left(k_{n} f\right)\left(f \in F^{+}\right)$. We define $k=\sum k_{n} \in F^{*+}$, and have $\psi(f)=\phi^{*}(k f)$. The finiteness of $k$ now follows from the lemma in 4.2.

$(2) \rightarrow(3)$. As before, it is easily seen that, without loss of generality, we may assume that $\phi$ is strict. The minimal full-valued extension $\phi^{*}$ of $\phi$ is now simply the standard $F^{\prime}$-integral on $F\left(E^{*}, \lambda^{*}\right)$ where $\left(E^{*}, \lambda^{*}\right)=(E, \lambda) \times E^{\prime}$. Let $\mu^{*}$ denote the standard relaxed $F^{\prime}$-measure on $(E, \mu) \times E^{\prime}$. For each $t \in E^{*}$ we define $\nu(t)=\mu^{*}\left(\xi^{-1}(t z)\right)$, where $\xi$ is the given isomorphism of $(E, \mu) \times E^{\prime}$ onto $E^{*}(z)$. Clearly $\nu$ is a relaxed $F^{\prime}$-measure on $E^{*}$; we assert that $[\nu(t)] \leqq\left[\lambda^{*}(t)\right]$ for all $t \in E^{*}$. For let $\left[\lambda^{*}(t)\right]=y^{\prime}$; then $t \leqq e \times y^{\prime}$, so 
$[\nu(t)] \leqq\left[\nu\left(e \times y^{\prime}\right)\right]=\left[\mu^{*}\left(\xi^{-1}\left(z\left(e \times y^{\prime}\right)\right)\right)\right]=\left[\mu^{*}\left(e \times y^{\prime}\right)\right] \leqq y^{\prime}$. Hence by Theorem 5 (4.3), since $\phi^{*}$ is full-valued, there exists a finite non-negative $k \in F^{*}$ such that, for all $t \in E^{*}, \nu(t)=\phi^{*}(k \chi(t))$. In particular, if $x \in E$ we have $\nu\left(x \times e^{\prime}\right)$ $=\phi^{*}\left(k \chi\left(x \times e^{\prime}\right)\right)$. But $\nu\left(x \times e^{\prime}\right)=\mu^{*}\left(x \times e^{\prime}\right)=\mu(x)$. Since $x$ corresponds to $x \times e^{\prime}$, in the natural imbedding of $E$ in $E^{*}$, this gives $\psi(f)=\phi^{*}(k f)$ whenever $f$ is a characteristic function in $F$, and so whenever $f \in F^{+}$.

$(3) \rightarrow(2)$. Again we may assume that $\phi$ is strict, and so have $F^{*}=F\left(E^{*}\right)$ where $E^{*}=(E, \lambda) \times E^{\prime}$ with standard $F^{\prime}$-measure $\lambda^{*}$. For each $t \in E^{*}$, write $\bar{\mu}(t)=\phi^{*}(k \chi(t))$; thus $\bar{\mu}$ is a relaxed $F^{\prime}$-measure on $E^{*}$. Define $z=[k]$; then if $t \in E^{*}(z)$ we have $\bar{\mu}(t) \neq 0$ unless $t=o$, so that $\bar{\mu}$ is a strict $F\left(E^{\prime \prime}\right)$-measure on $E^{*}(z)$, where $E^{\prime \prime}$ is the principal ideal of $[\bar{\mu}(z)]=\left[\phi^{*}(k)\right]=\left[\lambda^{*}(z)\right]$ in $E^{\prime}$.

Now let $\mu_{1}$ denote the strict form of the relaxed $F^{\prime}$-measure $\mu$ corresponding to $\psi ; \mu_{1}$ will be an $F_{1}^{\prime}$-measure on $E_{1}$, say, where $E_{1}$ is a certain principal ideal in $E$, and where $F_{1}^{\prime}=F\left(E^{\prime}[\mu(e)]\right)$. Since $[\mu(e)]=\left[\phi^{*}\left(k \chi\left(e \times e^{\prime}\right)\right)\right]$ $=\left[\lambda^{*}(z)\right]$, we have $F_{1}^{\prime}=F\left(E^{\prime \prime}\right)$.

We shall set up an isomorphism between $\left(E_{1}, \mu_{1}\right) \times E^{\prime \prime}$, with standard $F_{1}^{\prime}$-measure say $\mu^{*}$, and $E^{*}(z)$ with $F_{1}^{\prime}$-measure $\bar{\mu}$. To do this, we first define a mapping $\xi$ of the "rectangles" $x \times y^{\prime}\left(x \in E_{1}, y^{\prime} \in E^{\prime \prime}\right)$, by setting $\xi\left(x \times y^{\prime}\right)$ $=z\left(x \times y^{\prime}\right)$ in $E^{*}$. Then $\bar{\mu}\left\{\xi\left(x \times y^{\prime}\right)\right\}=\phi^{*}\left\{k \chi(z) \chi\left(x \times y^{\prime}\right)\right\}=\phi^{*}\left\{k \chi\left(x \times y^{\prime}\right)\right\}$ $=\phi^{*}\left(k \chi\left(x \times e^{\prime}\right)\right) \chi\left(y^{\prime}\right)=\psi(\chi(x)) \chi\left(y^{\prime}\right)$ (since $\chi\left(x \times e^{\prime}\right)$ is the function on $E^{*}$ which corresponds to $\chi(x) \in F$ in the natural imbedding of $F$ in $F\left(E^{*}\right)$ ), and therefore $\bar{\mu}\left\{\xi\left(x \times y^{\prime}\right)\right\}=\mu^{*}\left(x \times y^{\prime}\right)$.

We next extend $\xi$, in an obvious way, to all finite unions of rectangles. To show that $\xi$, as so extended, is $1-1$, we note that (by considering the symmetric difference) it suffices to prove that if a finite union of rectangles in $E^{*}$ meets $z$ in a null set (in $\left.E^{*}\right)$, then that union of rectangles is null in $\left(E_{1}, \mu_{1}\right)$ $\times E^{\prime \prime}$; and this follows immediately from the above relation between $\bar{\mu}$ and $\mu^{*}$. Thus $\xi$ is now an $F_{1}^{\prime}$-measure-preserving (finitely additive) isomorphism between the finitely additive algebras generated by the rectangles in $\left(E_{1}, \mu_{1}\right)$ $\times E^{\prime \prime}$, and their intersections with $z$ in $E^{*}$. By Theorem 1 (3.3), $\xi$ can be extended to a $\sigma$-isomorphism (still denoted by $\xi$ ) between the Borel fields so generated. Since $\left(E_{1}, \mu_{1}\right) \times E^{\prime \prime}$ is the same as $(E, \mu) \times E^{\prime}, \xi$ gives a $\sigma$-isomorphism of $(E, \mu) \times E^{\prime}$ onto $E^{*}(z)$, of the kind required by the theorem.

5.5 COROLlary. With the same notation as in Theorem 7 , the statement that $\phi$ dominates $\psi$ is also equivalent to each of the following statements:

(4) For every extension $\widetilde{\phi}$ of $\phi$, say to $\widetilde{F}$, there exists an extension $\tilde{\psi}$ of $\psi$ to $\tilde{F}$ such that $\tilde{\phi}$ dominates $\tilde{\psi}$.

(5) For every full-valued extension $\phi^{*}$ of $\phi$, say to $F^{*}$, there exists an extension $\psi^{*}$ of $\psi$ to $F^{*}$ which has a kernel representation in terms of $\phi^{*}$.

(6) There exist extensions $\phi^{*}$ and $\psi^{*}$, of $\phi$ and $\psi$ respectively, say to $F^{*}$, such that $\phi^{*}$ is full-valued and $\left[\psi^{*}(f)\right] \leqq\left[\phi^{*}(f)\right]$ for all $f \in F^{*+}$.

(7) $\psi$ has an "extended kernel representation" in terms of $\phi$; that is, for at 
least one extension $\phi^{*}$ of $\phi$, say to $F^{*}$, there is a finite $k \in F^{*+}$ such that $\psi(f)$ $=\phi^{*}(k f)$ whenever $f \in F^{+}$.

Proof. Suppose $\phi$ dominates $\psi$; we deduce (4). Given the extension $\tilde{\phi}$ of $\phi$ to $\widetilde{F}$, we extend $\widetilde{\phi}$ to a full-valued relaxed $F^{\prime}$-integral, say $\widetilde{\phi}^{*}$ on $\widetilde{F}^{*}$ (cf. Theorem $4,4.1$ ). Then, by Theorem $4, \tilde{\phi}^{*}$ is also an extension of the minimal fullvalued extension $\phi^{*}$ of $\phi$, say to $F^{*}$; and we may suppose $F \subset F^{*} \subset \widetilde{F}^{*}$. By Theorem $7(3)$, there exists a finite $k \in F^{*+} \subset \tilde{F}^{*+}$ such that $\psi(f)=\phi^{*}(k f)$ $=\tilde{\phi}^{*}(k f)$ whenever $f \in F^{+}$. Define $\tilde{\psi}$ on $\tilde{F}$ by: $\tilde{\psi}(f)=\tilde{\phi}^{*}(k f)\left(f \in \tilde{F}^{+}\right)$. Clearly $\tilde{\psi}$ is an extension of $\psi$; and $\tilde{\phi}$ dominates $\tilde{\psi}$ by the argument in 5.3.

The implications $(4) \rightarrow(5) \rightarrow(6)$ follow from the lemma in 5.3 , and $(6) \rightarrow(7)$ is immediate from Theorem 5 (4.3). Finally, to show that if (7) holds then $\phi$ dominates $\psi$, we write $k=\sum k_{n}(n=1,2, \cdots)$ where $k_{n} \in F^{*+}$ and is bounded, say $0 \leqq k_{n} \leqq \rho_{n}$, and define $\psi_{n}(f)=\phi^{*}\left(k_{n} f\right)\left(f \in F^{+}\right)$; clearly $\psi_{n}$ is a relaxed $F^{\prime}$-integral on $F, \sum \psi_{n}=\psi$, and $\psi_{n}(f) \leqq \phi^{*}\left(\rho_{n} f\right)=\rho_{n} \phi(f)\left(f \in F^{+}\right)$.

5.6 Mutual domination. It is easy to deduce, from Theorem 7 and the above corollary to it, necessary and sufficient conditions for each of two relaxed $F^{\prime}$-integrals to dominate the other. Here we state only the more significant results, omitting the proofs.

THEOREM 7'. With the same notation as in Theorem 7, each of the following statements is equivalent to the statement that $\phi$ and $\psi$ dominate each other.

(1) There exist relaxed $F^{\prime}$-integrals $\phi_{n}, \psi_{n}$, on $F$, and positive (finite) constants $\rho_{n}, \sigma_{n}\left(\sigma_{n}<\rho_{n}\right)$, such that (for all $\left.f \in F^{+}\right) \quad \sum \phi_{n}(f)=\phi(f), \sum \psi_{n}(f)=\psi(f)$, and $\sigma_{n} \phi_{n}(f) \leqq \psi_{n}(f) \leqq \rho_{n} \phi_{n}(f)$.

(2) $(E, \lambda) \times E^{\prime}$ and $(E, \mu) \times E^{\prime}$ are (algebraically) isomorphic in such a way that, for all $x \in E$ and $y \in E^{\prime}$, the element $x \times y^{\prime}$ corresponds to itself.

(3) If $\phi^{*}$ is any full-valued extension of $\phi$, say to $F^{*}$, there exist a full-valued extension $\psi^{*}$ of $\psi$ to $F^{*}$, and an element $k \in F^{*+}$ such that $0 \ll k \ll \infty$, such that $\psi^{*}(f)=\phi^{*}(k f)$ for all $f \in F^{*+}$.

(4) There exist full-valued extensions $\phi^{*}, \psi^{*}$ of $\phi$ and $\psi$ to the same function space $F^{*}$, such that $\left[\phi^{*}(f)\right]=\left[\psi^{*}(f)\right]$ for all $f \in F^{*+}$.

5.7 Some examples. The following examples show that there are, in general, no further relations between the properties discussed in 5.3.

(a) Let $E$ be the algebra of measurable sets mod null sets on the interval $0 \leqq y \leqq 1$, and let $E^{\prime}$ similarly consist of the measurable sets mod null sets on $0 \leqq x \leqq 1$. Define mappings $\phi, \psi$, of $F(E)+$ in $F\left(E^{\prime}\right)+$ by:

$$
\begin{array}{lll}
\phi(f)=g \quad \text { where } \quad g(x)=\int_{0}^{1} f(y) d y \quad \text { (a constant function), } \\
\psi(f)=h \text { where } h(x)=\frac{1}{x} \int_{0}^{1} f(y) d y .
\end{array}
$$

Both $\phi$ and $\psi$ are strict $F^{\prime}$-integrals on $F$, where $F^{\prime}=F\left(E^{\prime}\right)$ and $F=F(E)$; 
and it is easy to see that each dominates the other. In fact, it follows easily from Theorem 2 (3.5) that both have their minimal full-valued extensions $\phi^{*}, \psi^{*}$, on the space $F^{*}$ of measurable functions ( $\bmod$ null sets) on the unit square $0 \leqq x, y \leqq 1$; we have $\phi^{*}\left(f^{*}\right)=g^{*}$ where $g^{*}(x)=\int_{0}^{1} f^{*}(x, y) d y$, and $\psi^{*}\left(f^{*}\right)=h^{*}$ where $h^{*}(x)=\int_{0}^{1}(1 / x) f^{*}(x, y) d y$, and the kernel giving $\psi^{*}$ in terms of $\phi^{*}$ is, of course, the function $1 / x$, which is finite and positive almost everywhere. (To see that $\phi^{*}$ and $\psi^{*}$ do extend $\phi$ and $\psi$, recall that the function $f \in F$ corresponds to the function $f^{*} \in F^{*}$ whose value $f^{*}(x, y)=f(y)$, independent of $x$.) Nevertheless, 5.3 (2) fails; that is, it is impossible to decompose the interval $0 \leqq y \leqq 1$ into a sequence of measurable sets $a_{n}$ such that $\mu(t) \leqq \rho_{n} \lambda(t)$ for all $t \leqq a_{n}$, where $\mu$ and $\lambda$ are the $F^{\prime}$. measures corresponding to $\phi$ and $\psi$. For, if $t \neq o, \mu(t) / \lambda(t)=1 / x>\rho_{n}$ if $x$ is small enough.

(b) With the same $E, E^{\prime}$, and $\phi$ as before, take $\psi(f)=h$ where $h(x)$ $=x \int_{1}^{0} f(y) d y$. Again $\phi$ and $\psi$ are strict $F^{\prime}$-integrals on $F$, and each dominates the other. The kernel representation for the extensions $\psi^{*}, \phi^{*}$ is as before, but with kernel $x$ instead of $1 / x$. Here 5.3 (2) is satisfied; but clearly $\psi$ has no kernel representation in terms of $\phi$-i.e., with kernel in $F$.

(c) In 4.4, an example was given, again with the same $E$ and $E^{\prime}$, of two (strict) $F^{\prime}$-integrals $\phi, \psi$, such that $[\phi(f)]=[\psi(f)]$ for all $f \in F^{+}$, but such that neither has a kernel representation in terms of the other. We now show that neither dominates the other, so that neither has an extended kernel representation in terms of the other. This does not seem to be easy directly, and we shall use Theorem 7. Using Theorem 2 (3.5), it is easy to see that the product $(E, \lambda) \times E^{\prime}$ is now simply the algebra $(H, \Lambda)$ of all measurable subsets of $H$, with $\Lambda(z)=$ the function of $x$ whose value at each $x$ is the linear measure of the section of $z$ over $x$. Similarly $(E, \mu) \times E^{\prime}$ consists of all measurable subsets of $K$, with corresponding section-measure $M$. We shall show that there is no "natural" $\sigma$-isomorphism between any nonzero principal ideals in $(H, \Lambda)$ and $(K, M)$ respectively. Here "natural" means that the isomorphism extends the obvious correspondence between "rectangles"; the isomorphism need not, of course, preserve the $F^{\prime}$-measures. For suppose that the ideals of $H_{1} \subset H$ and $K_{1} \subset K$ were "naturally" isomorphic. Let $m$ denote ordinary plane measure; take $\epsilon>0$ such that $\epsilon<m\left(H_{1}\right)$, and cover $K_{1}$ by a sequence of rectangles $r_{n}$ such that $\sum m\left(r_{n}\right)<m\left(K_{1}\right)+\epsilon$. Let $s=H_{1}-U r_{n}$; then $m(s)>0$ (for $\left.H_{1} \cap K_{1}=0\right)$, and so $\Lambda(s)>0$ on a set of positive measure. Hence $s \neq 0$ in $\left(H_{1}, \Lambda\right)$. But under the supposed isomorphism, $H_{1} \cap r_{n}$ corresponds to $K_{1} \cap r_{n}$, and therefore $H_{1}-r_{n}$ corresponds to $K_{1}-r_{n}$, so that the image of $s$ is contained in $\cap\left(K_{1}-r_{n}\right)=K_{1}-U r_{n}=0$, giving a contradiction. From Theorem 7 (2) (5.4) it now follows that neither $\phi$ nor $\psi$ dominates the other.

\subsection{Simultaneous domination.}

THEOREM 8. Given a sequence of relaxed $F^{\prime}$-integrals $\psi_{n}$ on $F(n=1,2, \cdots)$, there exists a relaxed $F^{\prime}$-integral $\Phi$ on $F$ such that (i) $\Phi$ dominates each $\psi_{n}$, (ii) $\Phi(1) \leqq 1$. 
This is an easy consequence of the following lemma:

Lemma. Given a relaxed $F^{\prime}$-integral $\psi$ on $F$, and given $\epsilon>0$, there exists a relaxed $F^{\prime}$-integral $\phi$ on $F$ which dominates $\psi$ and satisfies $\phi(1) \leqq \epsilon$.

In fact, once the lemma is established, we obtain for each $n$ a relaxed $F^{\prime}$-integral $\phi_{n}$ on $F$ which dominates $\psi_{n}$ and satisfies $\phi_{n}(1) \leqq 1 / 2^{n}$. Define $\Phi=\sum \phi_{n}$; that is, $\Phi(f)=\sum \phi_{n}(f)$ for each $f \in F^{+}$. It is easy to see that $\Phi$ is a relaxed $F^{\prime}$-integral on $F$, and dominates each $\psi_{n}$; and clearly $\Phi(1) \leqq 1$.

To prove the lemma, let $\mu$ be the relaxed $F^{\prime}$-measure on $E$ which corresponds to $\psi$; by $2.2(\mathrm{~b})$, we can write $e=\sup a_{n}(n=1,2, \cdots)$ where the elements $a_{n}$ are disjoint and $\mu\left(a_{n}\right) \ll \infty$. For each $n$, let $b_{n m}$ denote the element of $E^{\prime}$ on which $m-1 \leqq \mu\left(a_{n}\right)<m$; thus $\sup _{m} b_{n m}=e^{\prime}$ and $\psi\left(\chi\left(a_{n}\right)\right) \chi\left(b_{n m}\right)$ $\leqq m \chi\left(b_{n m}\right)$. Define relaxed $F^{\prime}$-integrals $\psi_{n m}, \phi_{n m}, \phi$ on $F$ by setting (for each $\left.f \in F^{+}\right) \quad \psi_{n m}(f)=\psi\left\{f \chi\left(a_{n}\right)\right\} \chi\left(b_{n m}\right), \quad \phi_{n m}(f)=\left(\epsilon / m 2^{n}\right) \psi_{n m}(f), \quad$ and $\phi=\sum \phi_{n m}$ $(m, n=1,2, \cdots)$. Clearly $\psi=\sum \psi_{n m}$, so that $\phi$ dominates $\psi$; and, since $\phi_{n m}(1) \leqq\left(\epsilon / 2^{n}\right) \chi\left(b_{n m}\right)$, we have $\phi(1) \leqq \sum\left(\epsilon / 2^{n}\right) \chi\left(e^{\prime}\right)=\epsilon$.

5.9 Simultaneous representation of relaxed $F^{\prime}$-integrals. Let $\psi_{1}, \psi_{2}, \cdots$ be a given sequence of relaxed $F^{\prime}$-integrals on $F$. The preceding theory enables us to represent them all simultaneously in the form of kernel integrals in a product space. To simplify the statement of the theorem, we write $e_{1}^{\prime}=\left[\sum \psi_{n}(1)\right], e_{1}=e-\sup \left\{x \mid \sum \psi_{n}(\chi(x))=0\right\}$, and use $E_{1}^{\prime}$ and $E_{1}$ to denote the respective principal ideals $E^{\prime}\left(e_{1}^{\prime}\right), E\left(e_{1}\right)$, of these elements in $E^{\prime}$ and $E$. To each $\psi_{n}$ there corresponds, in a natural way, a relaxed $F\left(E_{1}^{\prime}\right)$-integral $\psi_{n}^{1}$ on $F\left(E_{1}\right)$; explicitly, the relation between $\psi_{n}$ and $\psi_{n}^{1}$ is that, for each $f \in F^{4}$,

$$
\psi_{n}(f)=\left\{\begin{array}{l}
\psi_{n}^{1}\left(f \chi\left(e_{1}\right)\right) \text { on } e_{1}^{\prime}, \\
0 \text { on } e^{\prime}-e_{1}^{\prime} .
\end{array}\right.
$$

THEOREM 9. With the above notation, $E_{1}$ is isomorphic to a subalgebra of a principal ideal in a direct product $(J, m) \times E_{1}^{\prime}$, where $(J, m)$ is a $\sigma$-finite nonatomic numerical measure algebra. This isomorphism can be so chosen that there exist finite non-negative functions $k_{n}(n=1,2, \cdots)$ on $(J, m) \times E_{1}^{\prime}$ such that, if $f \in F\left(E_{1}\right)+$ corresponds to $f^{*} \in F\left\{(J, m) \times E_{1}^{\prime}\right\}+$ under the isomorphism, we have

$$
\psi_{n}^{1}(f)=\int k_{n} f^{*} d M
$$

the last integral here denoting the standard integral on $F\left\{(J, m) \times E_{1}^{\prime}\right\}$. Modulo null sets, its value at each point $s$ of $E_{1}^{\prime}$ is the ordinary Lebesgue integral $\int_{J} k_{n}(x, s) f^{*}(x, s) d m(x)[6$, p. 165].

Proof. There is no loss of generality in assuming $e_{1}^{\prime}=e^{\prime}$ and $e_{1}=e$; thus $\psi_{n}^{1}=\psi_{n}$. By Theorem 8 , there is a relaxed $F^{\prime}$-integral $\Phi$ on $F$ which dominates every $\psi_{n}$; and $\Phi$ must now be a strict $F^{\prime}$-integral. By $[6$, Theorem 8, p. 172] 
applied to the $F^{\prime}$-integral $\Phi, E$ is isomorphic to a subalgebra of a principal ideal in a product $(J, m) \times E^{\prime}$ of the kind specified in Theorem 9 , in such a way that $\Phi$ is extended by the standard integral (say) $\Phi^{*}$ on this product. Now $\Phi^{*}$ is full-valued; hence, by the corollary to Theorem 7 (5.5 (5)), there exists for each $n$ a finite $k_{n} \in F\left\{(J, m) \times E^{\prime}\right\}+$ such that $\psi_{n}(f)=\Phi^{*}\left(k_{n} f^{*}\right)$ (for all $f \in F^{+}$), where $f^{*}$ is the element of $F\left\{(J, m) \times E^{\prime}\right\}$ which corresponds to $f \in F$ under the isomorphism of $E$. That is, $\psi_{n}(f)=\int k_{n} f^{*} d M$.

6. $F^{\prime}$-operators of bounded variation.

6.1 Let $F=F(E)$ and $F^{\prime}=F\left(E^{\prime}\right)$ be two function spaces (possibly the same). An " $F$ '-operator of bounded variation," or $F^{\prime}$-operator for short, on $F$, is to be a suitable mapping $\phi$ of a suitable subset $G$ of $F$, in $F^{\prime}$. We require $G$ to satisfy the following three conditions (which are certainly satisfied if $G$ consists of all finite functions in $F$ ):

(I) $G$ is a real linear space; i.e., if $g, h \in G$ and $\alpha, \beta$ are real (and finite), $\alpha g+\beta h$ exists and belongs to $G$.

(II) If $g \in G, f \in F$, and $|f| \leqq|g|$, then $f \in G$.

(III) There exists a sequence of elements $h_{n} \in G^{+}$such that

$$
\sum h_{n} \gg 0 \text {. }
$$

It is easy to see that, in the presence of (I) and (II), (III) is equivalent to:

(III) ${ }^{\prime}$ There exists a sequence of elements $a_{n} \in E$ such that $\sup a_{n}=e$ and $\chi\left(a_{n}\right) \in G(n=1,2, \cdots)$.

In view of $[6$, Lemma 1, p. 157], we now have:

(1) Given any $f \in F^{+}$, there exist elements $g_{n} \in G^{+}(n=1,2, \cdots)$ such that $\sum g_{n}=f$.

Now let $\phi$ be a mapping of $G$ in $F^{\prime}$ such that

(IV) $\phi$ is linear; i.e., whenever $g, h \in G$ and $\alpha, \beta$ are real, then $\alpha \phi(g)+\beta \phi(h)$ exists and equals $\phi(\alpha g+\beta h)$.

(V) If $g_{n} \in G^{+}$and $\sum g_{n} \in G(n=1,2, \cdots)$, then

$$
\phi\left(\sum g_{n}\right)=\sum \phi\left(g_{n}\right) \text {. }
$$

We define the "variation" $V_{\phi}$ of $\phi$ to be the mapping of $F^{+}$in $F^{\prime}$ given by

$$
V_{\phi}(f)=\sup \left\{\sum\left|\phi\left(g_{n}\right)\right|\right\}
$$

the supremum being taken over all decompositions $f=\sum g_{n}(n=1,2, \cdots)$ where $g_{n} \in G^{+}$. (Such decompositions exist, from (1).) We note that:

(3) If $g \in G$, then $|g| \ll \infty,|\phi(g)| \ll \infty$, and $|\phi(g)| \leqq V_{\phi}(|g|)$.

For if $g$ were somewhere infinite, $1 g+(-1) g$ would not exist, violating (I); and the finiteness of $\phi(g)$ follows similarly from (IV). The remaining assertion is immediate from (IV) and the decomposition $|g|=g^{+}+g^{-}$.

If further

$$
V_{\phi}(g) \ll \infty \text { whenever } g \in G^{+},
$$


$\phi$ will be called an $F^{\prime}$-operator of bounded variation on $F$, or simply " $F^{\prime}$-operator on $F$," for short; and $G$ is called the "domain" of $\phi$.

6.2 An $F^{\prime}$-operator $\phi$ is said to be positive if it maps $G^{+}$in $F^{\prime+}$-i.e., if $\phi(g) \geqq 0$ whenever $g \in G$ and $g \geqq 0$. For a positive $F^{\prime}$-operator, clearly $V_{\phi}(f)$ $=\phi(f)$ whenever $f \in G^{+}$, so that (VI) is now superfluous. By the argument in [6, p. 163], a positive $F^{\prime}$-operator $\phi$ can always be extended (uniquely) to all of $F^{+}$so as to become a relaxed $F^{\prime}$-integral on $F\left(^{9}\right)$. Conversely, it is easy to see that if $\psi$ is a relaxed $F^{\prime}$-integral on $F$, and if $G$ is the set of all $f \in F$ satisfying: $\psi(f)$ is defined, and $f$ and $\psi(f)$ are both finite, then $\psi$ restricted to $G$ is a positive $F^{\prime}$-operator on $F$, with $G$ as its domain. Thus "positive $F^{\prime}$-operator" and "relaxed $F^{\prime}$-integral" are substantially equivalent notions.

6.3 Most of the elementary theory of real functions of bounded variation of a real variable extends to $F^{\prime}$-operators; we shall sketch the theory, giving details only when the generalization is nontrivial. In the first place, every $F^{\prime}$-operator is the difference between two positive $F^{\prime}$-operators, and conversely $\left({ }^{10}\right)$. More precisely:

Theorem 10. Let $P, Q$ be any positive $F^{\prime}$-operators on $F$, with domains $G, H$, and let $\psi(f)=P(f)-Q(f)(f \in G \cap H)$; then $\psi$ is an $F^{\prime}$-operator with domain $G \cap H$, and $V_{\psi}(f) \leqq P(f)+Q(f)\left(f \in(G \cap H)^{+}\right)$. Conversely, given an $F^{\prime}$-operator $\phi$ with domain $G$, define $G^{*}=\left\{f|f \in F| f \mid, \ll \infty\right.$, and $\left.V_{\phi}(|f|) \ll \infty\right\}$; then $G^{*} \supset G$, and there exist positive $F^{\prime}$-operators $P, Q$, each with domain $G^{*}$, such that (i) $P(g)-Q(g)=\phi(g)$ whenever $g \in G$, (ii) $P(g)+Q(g)=V_{\phi}(g)$ whenever $g \in G^{*+}$; and further these conditions determine $P$ and $Q$ uniquely.

The direct assertion of the theorem is easily verified. To prove the converse assertion, we use (1) to define, for all $f \in F^{+}$,

$$
P(f)=\sup \left\{\sum\left(\phi\left(g_{n}\right)\right)^{+}\right\}, \quad Q(f)=\sup \left\{\sum\left(\phi\left(g_{n}\right)\right)^{-}\right\},
$$

both suprema being taken over all decompositions $f=\sum g_{n}$ where $g_{n} \in G^{+}$. Trivially $V_{\phi}(f) \leqq P(f)+Q(f) \quad\left(f \in F^{+}\right)$. But, given any two decompositions $f=\sum g_{i}=\sum h_{j}$, where $g_{i}, h_{j} \in G^{+}(i, j=1,2, \cdots)$, one can find elements $k_{i j} \in F^{+}$such that $\sum_{j} k_{i j}=g_{i}$ and $\sum_{i} k_{i j}=h_{j}$; and, from (II), $k_{i j} \in G^{+}$. Thus, using (V), we obtain

$$
\begin{aligned}
\sum\left(\phi\left(g_{i}\right)\right)^{+}+\sum\left(\phi\left(h_{j}\right)\right)^{-} & \leqq \sum \sum\left(\phi\left(k_{i j}\right)\right)^{+}+\sum \sum\left(\phi\left(k_{i j}\right)\right)^{-} \\
& =\sum \sum\left|\phi\left(k_{i j}\right)\right| \leqq V_{\phi}(f),
\end{aligned}
$$

(9) The postulates (i) and (v) of the quoted lemma need not be satisfied here, but are needed only for the "normalizing" postulates $(\gamma),(\delta)$.

(10) Thus the $F^{\prime}$-operators (of bounded variation) roughly coincide with the "regular operators" of [2] and [3]. The differences are: (i) a regular operator is defined on the whole of a given conditionally complete vector lattice $L$, (ii) $L$ need not have a unit, nor satisfy the countable chain condition, though it is in fact expressible as a direct sum of ideals $L_{\alpha}$ in such a way that, restricted to each $L_{\alpha}$, the regular operator can be regarded as an $F^{\prime}$-operator of the above type, and (iii) $L$ is subjected to further requirements, which are not needed here. 
from which it follows that $P(f)+Q(f) \leqq V_{\phi}(f)$. Thus we have proved

$$
P(f)+Q(f)=V_{\phi}(f)
$$

$\left(f \in F^{+}\right)$.

Again, suppose $g \in G^{+}$and $g=\sum g_{i}$ where $g_{i} \in G^{+}$; then $\sum\left(\phi\left(g_{i}\right)\right)^{+}$ $=\sum\left\{\phi\left(g_{i}\right)+\left(\phi\left(g_{i}\right)\right)^{-}\right\}=\sum \phi\left(g_{i}\right)+\sum\left(\phi\left(g_{i}\right)\right)^{-} \leqq \phi(g)+Q(g)$ (all the terms occurring here being finite, from (3)), so that $P(g) \leqq \phi(g)+Q(g)$. Similarly $Q(g) \leqq-\phi(g)+P(g) ;$ hence

$$
\phi(g)=P(g)-Q(g)
$$$$
\left(g \in G^{+}\right) \text {. }
$$

Next we show that

$$
P, Q \text {, and } V_{\phi} \text { are } \sigma \text {-additive on } F^{+} \text {. }
$$

For if $f=\sum f_{n}$, where $f_{n} \in F^{+}$, then for any decompositions $f_{n}=\sum_{m} g_{n m}$ $\left(g_{n m} \in G^{+}\right)$we have

$$
P(f) \geqq \sum \sum\left(\phi\left(g_{n m}\right)\right)^{+} \geqq \sum_{n=1}^{N}\left\{\sum_{m=1}^{\infty}\left(\phi\left(g_{n m}\right)\right)^{+}\right\} .
$$

Keeping $N$ fixed, we obtain $P(f) \geqq \sum_{1}^{N} P\left(f_{n}\right)$, and so $P(f) \geqq \sum_{n=1}^{\infty} P\left(f_{n}\right)$. The reverse inequality follows by an argument similar to that proving (5), and this establishes (7) for $P$. The proof for $Q$ is similar, and the assertion for $V_{\phi}$ then follows from (5).

Using (7), one readily verifies that $G^{*}$ satisfies conditions (I) and (II) above; and, since clearly $G^{*} \supset G$, it follows that $G^{*}$ satisfies (III) also.

Now (VI) and (5) show that the functions $h_{n}$ in (III) satisfy $P\left(h_{n}\right) \ll \infty$. This, with (7), shows that $P$ is a relaxed $F^{\prime}$-integral, which (from 6.2) gives rise to a positive $F^{\prime}$-operator (still denoted by $P$ ) with domain (say) $H$. Similarly from $Q$ we obtain a positive $F^{\prime}$-operator, still denoted by $Q$, with domain $K$. It is easy to see that $H \cap K \supset G^{*}$ here, for $H$ consists of all finite $f \in F$ such that $P\left(f^{+}\right)$and $P\left(f^{-}\right)$are finite, and similarly for $K$. The relation (6) is easily seen to apply now to all $f \in G$. Finally, the uniqueness of $P$ and $Q$ is an immediate consequence of the following easily proved remark:

(8) If two $F^{\prime}$-operators $\psi_{1}, \psi_{2}$ agree on $G^{+}$, where $G$ is any operator domain (satisfying (I)-(III) above), they agree whenever both are defined.

6.4 Corollaries.

(1) Any $F^{\prime}$-operator $\phi$ can be extended uniquely to an $F^{\prime}$-operator $\phi^{*}$ with domain $G^{*}$, where $G^{*}=\left\{f|f \in F| f \mid, \ll \infty, V_{\phi}(|f|) \ll \infty\right\} ;$ and $G^{*}$ is the largest domain to which $\phi$ can be extended.

To extend $\phi$, merely define $\phi^{*}(f)=P(f)-Q(f)\left(f \in G^{*}\right)$. If $\phi^{\prime}$ is any extension of $\phi$ with domain $G^{\prime} \supset G$, we first note that $V_{\phi}(g)=V_{\phi^{\prime}}(g)$ if $g \in G^{+}$, since in any decomposition $g=\sum g_{n}$ where $g_{n} \in G^{\prime+}$ we have $g_{n} \in G$ from (II), so that $\phi\left(g_{n}\right)=\phi^{\prime}\left(g_{n}\right)$. Now, given $g^{\prime} \in G^{\prime}$, we use (1) to write $\left|g^{\prime}\right|=\sum g_{n}$ with $g_{n} \in G^{+}$, and have (from (7)) $V_{\phi}\left(\left|g^{\prime}\right|\right)=\sum V_{\phi}\left(g_{n}\right)=\sum V_{\phi^{\prime}}\left(g_{n}\right)=V_{\phi^{\prime}}\left(\left|g^{\prime}\right|\right)$ $\ll \infty$. Thus $\left|g^{\prime}\right| \in G^{*}$, whence $g^{\prime} \in G^{*}$, proving $G^{\prime} \subset G^{*}$. 
Definition. In accordance with this corollary, we call $G^{*}$ the maximal domain of the $F^{\prime}$-operator $\phi$.

(2) For any $F^{\prime}$-operator $\phi$ on $F$, with domain $G, V_{\phi}$ can be extended uniquely to a positive $F^{\prime}$-operator $V$; the maximal domain of $V$ is the same as that of $\phi$, and consists of $\{h|h \in F| h,|\ll \infty| V,(h) \mid \ll \infty\}$.

The required extension is obtained by defining $V(f)=P(f)+Q(f)\left(f \in G^{*}\right)$ $P$ and $Q$ being regarded as relaxed $F^{\prime}$-integrals. The uniqueness of the extension to $G^{*}$ follows from (8), and the statements about the maximal domain of $V$ are easily verified.

(3) ("Dominated convergence theorem") Let $\phi$ be an $F^{\prime}$-operator with domain $G$, and suppose $\left|f_{n}\right| \leqq g(n=1,2, \cdots)$, where $f_{n}, g \in G$. Then if $f_{n} \rightarrow f$ we have $f \in G$ and $\phi\left(f_{n}\right) \rightarrow \phi(f)$.

Suppose first that $f_{n} \in G^{+}$and that $f=0$. Write $g_{n}=\sup \left\{f_{m} \mid m \geqq n\right\}$; then $g_{1} \geqq g_{2} \geqq \ldots \geqq 0, g_{n} \in G$ (since $g_{n} \leqq g$ ), and $g_{n} \rightarrow 0$ (in the sense we use throughout, of pointwise convergence $\bmod \mathcal{N})$. The series $\left(g_{1}-g_{2}\right)+\left(g_{2}-g_{3}\right)+\cdots$ converges to $g_{1} \in G$; hence 6.3 (7) gives that $\sum V_{\phi}\left(g_{n}-g_{n+1}\right)$ converges to $V_{\phi}\left(g_{1}\right) \ll \infty$. Thus $\sum_{n \geqq m}\left\{V_{\dot{\phi}}\left(g_{n}\right)-V_{\phi}\left(g_{n+1}\right)\right\} \rightarrow 0$ as $n \rightarrow \infty$; that is, $V_{\phi}\left(g_{n}\right) \rightarrow 0$. A fortiori $V_{\phi}\left(f_{n}\right) \rightarrow 0$, and so $\phi\left(f_{n}\right) \rightarrow 0$. The general case now follows if we replace $f_{n}$ by $\left|f_{n}-f\right|$.

6.5 The Hahn decomposition. The positive $F^{\prime}$-operator $V_{\phi}$ can (as in 6.2) be regarded as a relaxed $F^{\prime}$-integral on $F$. If it is full-valued (as is certainly the case if $F^{\prime}$ consists of the real numbers, $E^{\prime}$ reducing to a single atom), the Hahn decomposition theorem for numerical functions of bounded variation has the following generalization.

THEOREM 11. If $\phi$ is an $F^{\prime}$-operator on $F(E)$, with domain $G$, for which $V_{\phi}$ is full-valued, there exists a decomposition $e=p \vee q$, where $p q=o$, such that for all $f \in G^{+}$we have $\phi(f) \geqq 0$ if $[f] \leqq p$, and $\phi(f) \leqq 0$ if $[f] \leqq q$.

That is, $\phi$ is a positive $F^{\prime}$-operator when restricted to the subspace $F(E(p))$, and $-\phi$ is positive when restricted to $F(E(q))$.

To prove the theorem, we use Theorem 10 to write $\phi=P-Q, V_{\phi}=P+Q$, where $P, Q$ are positive $F^{\prime}$-operators on $F=F(E)$, which can therefore be regarded as relaxed $F^{\prime}$-integrals. Now for all $f \in F^{+}$we have $0 \leqq P(f) \leqq V_{\phi}(f)$, and so $[P(f)] \leqq\left[V_{\phi}(f)\right]$; and a similar argument applies to $Q$. Hence, by Theorem 5 (4.3), there exist finite non-negative functions $h, k \in F$ such that $P(f)=V_{\phi}(h f)$ and $Q(f)=V_{\phi}(k f)$ for all $f \in F^{+}$. Hence $\phi(f)=V_{\phi}(g f)$ where $g=h-k$; and we have only to define (using any realization of $E$ ) $p$ to be the set where $g \geqq 0$, and $q=$ complement of $p$.

REMARK. If $V$ is not full-valued, the assertion of Theorem 11 may fail; e.g., it fails if $\phi$ is the $F^{\prime}$-integral on the space of measurable functions (mod null sets) of a real variable, given by: $\phi(f)=g^{\prime}$ where $g^{\prime}(x)=(\sin x) \int f(y) d y$. But in any case we obtain the following corollary:

If $\phi$ is an arbitrary $F^{\prime}$-operator (of bounded variation) on $F(E)$, there exists 
an extension of $\phi$, say to $\phi^{*}$ on $F\left(E^{*}\right)$ where $E^{*} \supset E$, and a decomposition $e^{*}=p^{*} \bigvee q^{*}$, where $p^{*} q^{*}=o$, such that $\phi^{*}$ is a positive $F^{\prime}$-operator when restricted to the subspace $F\left(E^{*}\left(p^{*}\right)\right)$, and $-\phi^{*}$ is a positive $F^{\prime}$-operator when restricted to $F\left(E^{*}\left(q^{*}\right)\right)$.

For $V_{\phi}$ has a minimal full-valued extension, say to $V^{*}$ on $F\left(E^{*}\right)$ (Theorem $4,4.1)$. By the corollary to Theorem $6(5.2)$ we can extend $P$ and $Q$ to $F\left(E^{*}\right)$ in such a way that the extended $F^{\prime}$-operators $P^{*}, Q^{*}$ satisfy $0 \leqq P^{*}(f) \leqq V^{*}(f)$, $0 \leqq Q^{*}(f) \leqq V^{*}(f)$ whenever $f \in F\left(E^{*}\right)^{+}$. We extend $\phi$ by setting $\phi^{*}=P^{*}-Q^{*}$; and the argument proving Theorem 11 now applies.

6.6 An illustration. As an illustration of the way in which $F^{\prime}$-operators occur, we consider a closed linear transformation $T$ on a real separable Hilbert space $\mathfrak{H}$; we regard $\mathfrak{H C}$ as consisting of the square-integrable functions on a measure space $(S, \mathcal{B}, \mathcal{N})$, where $\mathscr{B}$ and $\mathcal{N}$ consist of the measurable and null sets with respect to a $\sigma$-finite numerical measure, and we suppose that the domain $D$ of $T$ is dense in $\mathfrak{C}$. We take $F=F^{\prime}=F(S, \mathscr{B}, \mathcal{N}) \supset \mathfrak{H}$, and assert that $T$ is the strong limit of a sequence of $F^{\prime}$-operators (of bounded variation) on $F$. More precisely:

THEOREM 12. There exist $F$-operators $T_{n}$ on $F(n=1,2, \cdots)$, each with domain $\mathcal{H}$, such that (i) for each $n, T_{n}(\mathcal{H C})$ is a finite-dimensional linear subspace of $\mathfrak{H}$, (ii) for each $f \in D,\left\|T_{n}(f)-T(f)\right\| \rightarrow 0$ as $n \rightarrow \infty$.

Proof. $T$ has an adjoint $T^{*}$ whose domain $D^{*}$ is dense in $\mathfrak{H C}$ (cf. $[7, \mathrm{p}$. $304])$, and if $f \in D$ and $g \in D^{*}$ we have $\left(f, T^{*} g\right)=(T f, g)$. Let $\left\{g_{n}\right\}$ be a complete orthonormal sequence of elements of $D^{*}$, and define (for all $f \in \mathfrak{H}$ ) $T_{n}(f)=\sum\left(f, T^{*} g_{i}\right) g_{i}(1 \leqq i \leqq n)$. Then $T_{n}$ is a bounded linear transformation of $\mathfrak{H C}$ onto the linear subspace of $\mathcal{H}$ spanned by $g_{1}, g_{2}, \cdots, g_{n}$. But if $f \in D$, $T_{n}(f)=\sum_{1}^{n}\left(T f, g_{i}\right) g_{i}$, and so (from Parseval's theorem) $\left\|T_{n}(f)-T(f)\right\| \rightarrow 0$. Finally, to show that $T_{n}$ is an " $F$-operator" in our sense, it is enough to exhibit it as the difference between two positive $F$-operators. Write $T^{*}\left(g_{i}\right)=g_{i}{ }^{*}$, and for each $f \in \mathcal{F}$ define

$$
\begin{array}{ll}
P_{n}(f)=\sum\left\{\left(1 f, g_{i}^{*+}\right) g_{i}^{+}+\left(f, g_{i}^{*-}\right) g_{i}^{-}\right\} & (1 \leqq i \leqq n), \\
Q_{n}(f)=\sum\left\{\left(f, g_{i}^{*-}\right) g_{i}^{+}+\left(f, g_{i+}^{*+}\right) g_{i}^{-}\right\} & (1 \leqq i \leqq n) .
\end{array}
$$

Then $P_{n}, Q_{n}$ are positive $F$-operators with domain $\mathfrak{F}$; and $P_{n}(f)-Q_{n}(f)$ $=T_{n}(f)$.

Corollary. Every closed linear transformation on a separable Hilbert space is the strong limit of a sequence of bounded linear transformations, each with finite-dimensional range.

We remark that if we write $k_{n}(x, y)=\sum g_{i}^{*}(y) g_{i}(x)(1 \leqq i \leqq n)$ in the preceding argument, we have for $T_{n}$ the "kernel representation" $T_{n}(f)(x)$ $=\int f(y) k_{n}(x, y) d y$. This illustrates the following theorem, which applies to an 
arbitrary sequence of $F^{\prime}$-operators on an arbitrary function space $F$ (satisfying, as always, the countable chain condition).

6.7 THEOREM 13. Let $\phi_{1}, \phi_{2}, \cdots$, be a given sequence of $F^{\prime}$-operators on $F=F(E)$, with domains $G_{1}, G_{2}, \cdots$, and suppose ${ }^{(11)}$ that $\sum V_{\phi_{n}}(1) \gg 0$, and that for each $f \in F^{+}$we have $\sum V_{\phi_{n}}(f)>0$ unless $f=0$. Then there is an isomorphism of $E$ onto a subalgebra of a principal ideal of a direct product $(J, m)$ $\times E^{\prime}$, where $(J, m)$ is a $\sigma$-finite non-atomic numerical measure algebra, with the property that there exist finite functions $k_{1}, k_{2}, \cdots$, on $(J, m) \times E^{\prime}$ such that, if $f \in F$ corresponds under the isomorphism to $f^{*} \in F\left\{(J, m) \times E^{\prime}\right\}$, we have $\phi_{n}(f)=\int k_{n} f^{*} d M=\int_{J} k_{n}(x, s) f^{*}(x, s) d m(x)$ whenever $f \in G_{n}(n=1,2, \cdots)$.

To prove Theorem 13 , we merely write $\phi_{n}=P_{n}-Q_{n}$ as in Theorem 10 (6.3), where $P_{n}$ and $Q_{n}$ can be regarded as relaxed $F^{\prime}$-integrals on $F$. Theorem 13 now follows from an application of Theorem 9 (5.9) to the sequence $\left\{P_{n}, Q_{n}\right\}$.

As was remarked in the introduction to this paper, every operator expressible in the kernel form $\phi(f)=\int k f d M$ is necessarily of bounded variation in the present sense; hence Theorem 13 gives, in a sense, a complete description of the structure of an arbitrary sequence of $F^{\prime}$-operators (of bounded variation). As was also remarked in the introduction, the analogous theorem for complex function spaces would follow by almost the same argument.

\section{REFERENCES}

1. P. Erdös and J. C. Oxtoby, Partitions of the plane into sets having positıve measure in every non-null measurable product set, Trans. Amer. Math. Soc. vol. 79 (1955) pp. 91-102.

2. L. Kantorovitch, Linear operations in semi-ordered spaces I, Rec. Math. (Mat. Sbornik) N.S. vol. 7 (49) (1940) pp. 209-284.

3. L. Kantorovitch and B. Vulich, Sur la représentation des opérations linéaires, Compositio Math. vol. 5 (1937-38) pp. 119-165.

4. D. Maharam, The representation of abstract measure functions, Trans. Amer. Math. Soc. vol. 65 (1949) pp. 279-330.

5. - Decompositions of measure algebras and spaces, Trans. Amer. Math. Soc. vol. 69 (1950) pp. 142-160.

6. - The representation of abstract integrals, Trans. Amer. Math. Soc. vol. 75 (1953) pp. $154-184$.

7. F. Riesz and B. Sz. Nagy, Leçons d'analyse fonctionelle, Budapest, 1952.

The University,

MANCHESTER, ENGLAND.

(11) These suppositions involve no essential loss of generality; without them we merely replace $E$ and $E^{\prime}$ by suitable principal ideals, as in Theorem 9 (5.9). 